\title{
FOXP3+ regulatory $T$ cell development and function require histone/protein deacetylase 3
}

\author{
Liqing Wang, ${ }^{1}$ Yujie Liu, ${ }^{1}$ Rongxiang Han, ${ }^{1}$ Ulf H. Beier, ${ }^{2}$ Tricia R. Bhatti, ${ }^{1}$ Tatiana Akimova, ${ }^{1}$ Mark I. Greene, ${ }^{3}$ \\ Scott W. Hiebert, ${ }^{4,5}$ and Wayne W. Hancock ${ }^{1}$ \\ 'Division of Transplant Immunology, Department of Pathology and Laboratory Medicine, and Biesecker Center for Pediatric Liver Diseases and 2Division of Nephrology, Department of Pediatrics, \\ Children's Hospital of Philadelphia and Perelman School of Medicine at the University of Pennsylvania, Philadelphia, Pennsylvania, USA. ${ }^{3}$ Department of Pathology and Laboratory Medicine, \\ Perelman School of Medicine at the University of Pennsylvania, Philadelphia, Pennsylvania, USA. ${ }^{4}$ Department of Biochemistry, Vanderbilt University School of Medicine, Nashville, Tennessee, USA. \\ ${ }^{5}$ Vanderbilt-Ingram Cancer Center, Nashville, Tennessee, USA
}

\begin{abstract}
Treg dysfunction is associated with a variety of inflammatory diseases. Treg populations are defined by expression of the oligomeric transcription factor FOXP3 and inability to produce IL-2, a cytokine required for T cell maintenance and survival. FOXP3 activity is regulated post-translationally by histone/protein acetyltransferases and histone/protein deacetylases (HDACs). Here, we determined that HDAC3 mediates both the development and function of the two main Treg subsets, thymus-derived Tregs and induced Tregs (iTregs). We determined that HDAC3 and FOXP3 physically interact and that HDAC3 expression markedly reduces I/2 promoter activity. In murine models, conditional deletion of Hdac3 during thymic Treg development restored Treg production of IL-2 and blocked the suppressive function of Tregs. HDAC3-deficient mice died from autoimmunity by 4-6 weeks of age; however, injection of WT FOXP3+ Tregs prolonged survival. Adoptive transfer of Hdac3deficient Tregs, unlike WT Tregs, did not control T cell proliferation in naive mice and did not prevent allograft rejection or colitis. HDAC3 also regulated the development of iTregs, as HDAC3-deficient conventional T cells were not converted into iTregs under polarizing conditions and produced large amounts of IL-2, IL-6, and IL-17. We conclude that HDAC3 is essential for the normal development and suppressive functions of thymic and peripheral FOXP3+ Tregs.
\end{abstract}

\section{Introduction}

Considerable insights into the manner by which $\mathrm{FOXP}^{+}$Tregs maintain immune homeostasis, and evidence of how their dysfunction contributes to the pathogeneses of various diseases, are now available (1-4). However, the translational and therapeutic implications of such data are less clear. Proposed applications for Treg therapies include the prevention of graft-versus-host disease and solid organ transplant rejection, and stabilization of disease in patients newly diagnosed with type 1 diabetes. However, progress has been slow, given that questions remain regarding the choice of protocols for optimal Treg expansion (5); their phenotypic stability after transfer and associated potential risks of disease exacerbation (6); and the considerable costs of such trials using cells that have a brief life span, such that repeated infusions may be required for even a modicum of clinical efficacy (7-9).

An important alternative to Treg-based therapy is the modulation of Treg functions by pharmacologic means. This approach potentially has a significantly broader range of applications than Treg therapy, since enhancement of Treg functions may be useful in the management of autoimmunity and transplantation, whereas dampening of Treg function has potential utility in the context of tumor therapy. Proof-of-principle data are now available for both types of manipulations. Thus, pan-histone/protein deacetylase

Conflict of interest: The authors have declared that no conflict of interest exists Submitted: May 15, 2014; Accepted: December 16, 2014.

Reference information: / Clin Invest. 2015;125(3):1111-1123. doi:10.1172/JCI77088. inhibitors (pan-HDACi) increase FOXP3 acetylation and DNA binding, enhance Treg production and suppressive activity, and have beneficial effects in the prevention and treatment of autoimmune disease and transplant rejection (10). Conversely, targeting of the histone acetyltransferase p300 can impair FOXP3 acetylation and Treg function while preserving $\mathrm{T}$ effector cell (Teff) responses and thereby promote immune responses in tumor-bearing hosts (11).

Pan-HDACi have documented utility for the treatment of certain tumors but are generally considered too toxic for nononcologic indications (12). This has stimulated investigations into which HDACs can be usefully targeted so as to promote Treg function, and which HDACs should be left untouched so as to perform their essential cellular functions. The 11 classical, zinc-dependent HDAC metalloenzymes are categorized as class I (HDAC1, HDAC2, HDAC3, and HDAC8), class IIa (HDAC4, HDAC5, HDAC7, and HDAC9), class IIb (HDAC6 and HDAC10), and class III (HDAC11). Research has shown that gene deletion or knockdown of at least two class IIa HDAC enzymes, HDAC7 and HDAC9, can increase Treg suppressive functions in vitro and in vivo (12-14). However, class IIa HDACs have very limited catalytic activity using conventional substrates and likely function primarily via protein-protein interactions (15). In particular, the $\mathrm{N}$ termini of class IIa HDACs can recruit the class I HDAC HDAC3 to form multimolecular suppressive complexes (16-19). We have analyzed the effects of HDAC3 targeting on Treg functions, reasoning that if significant effects were seen, this might represent an important pharmacologic target for modulation of Treg functions. 
A

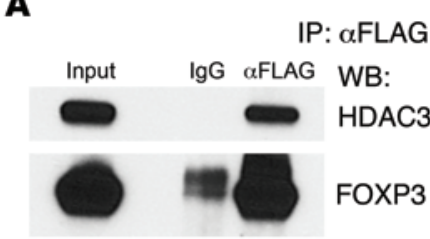

B

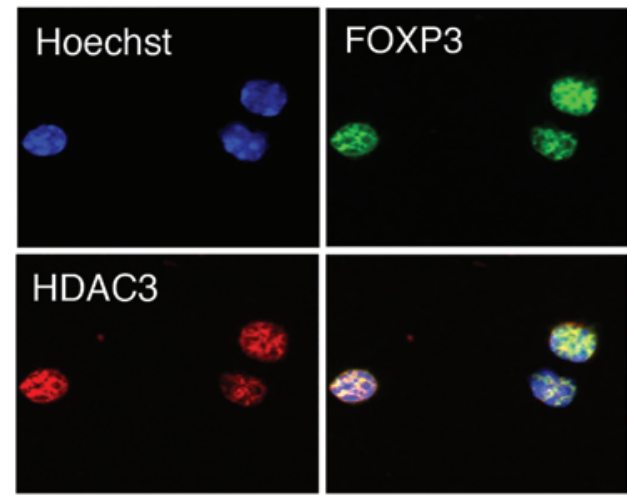

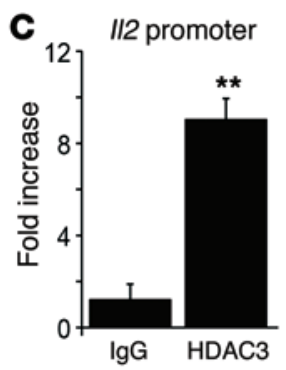

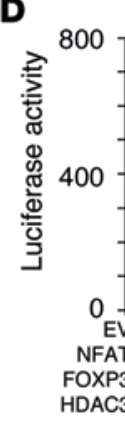

E

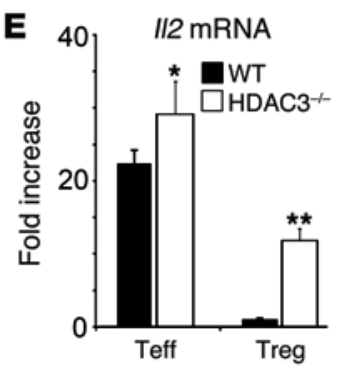

Figure 1. HDAC3 is required for suppression of IL-2 production in Tregs. (A) Co-immunoprecipitation of $\mathrm{HDAC} 3$ and $\mathrm{FOXP3}$ proteins from 293T cells transfected with FLAG-tagged HDAC3 and FOXP3 constructs. (B) Colocalization of HDAC3 and FOXP3 within the nuclei of WT Tregs (original magnification, $\times 400$ ). (C) ChIP assay showing recruitment of $\mathrm{HDAC} 3$ to the II2 promoter. (D) II2 promoter-driven luciferase activity in 293T cells transfected with empty vector (EV) or vectors for NFAT, FOXP3, and $H D A C 3$, as shown. (E) I/2 gene expression ( $\mathrm{qPCR}$ ) in conventional Teffs and Tregs. In $\mathbf{C}-\mathbf{E}$, mean $\pm S D, n=4$ /group, with statistical analysis using Student's $t$ test for unpaired data in $\mathbf{C}$ and $\mathbf{E}$, and Kruskal-Wallis with Dunn's multiple comparison test for $\mathbf{D} ;{ }^{*} P<0.05$ and ${ }^{*} P<0.01$ for the indicated comparisons, and versus WT in $\mathbf{E}$.

\section{Results}

HDAC3 co-associates with FOXP3 and suppresses IL-2 production in Tregs. FOXP3 plays a key role in Treg development and functions by controlling the expression of several hundred target genes that collectively determine the phenotype and suppressive activity of Tregs (20). Since HDAC3 is a key component of many suppressive complexes, we tested whether FOXP3 could physically interact with HDAC3. After transfection of 293T cells with Flag-tagged HDAC3 and FOXP3, cells were lysed and proteins immunoprecipitated with anti-Flag (HDAC3) antibody and probed with antimouse FOXP3 and anti-Flag antibodies. These studies showed that FOXP3 and HDAC3 can co-associate (Figure 1A). Consistent with this, confocal imaging confirmed the colocalization of FOXP3 and HDAC3 within the nuclei of murine Tregs (Figure 1B). We next sought a possible function of this interaction. A prime characteristic of Tregs is their inability to produce IL-2, as a result of suppression by FOXP3. Using ChIP assays, we found that HDAC3 binds to the $I l 2$ promoter in Tregs (Figure 1C). Moreover, using 293T cells expressing an NFAT-driven IL-2 luciferase construct, we found that $I l 2$ transcription was downregulated in cells transfected with FOXP3 and further inhibited by cotransfection with HDAC3, whereas HDAC3 expression in the absence of FOXP3 had no effect (Figure 1D). Inhibition of $I l 2$ gene transcription was not due to an effect of FOXP3 or HDAC3 transfection on NFAT expression (Supplemental Figure 1; supplemental material available online with this article; doi:10.1172/JCI77088DS1). Consistent with data from transfected cells, HDAC3 ${ }^{-/-}$Tregs, described below, had increased Il2 gene expression (Figure 1E). These studies indicate that HDAC3 can bind to FOXP3 and inhibit Treg production of IL-2.

Conditional deletion of HDAC3 within $\mathrm{FOXP}^{+}$Tregs results in lethal autoimmunity. As HDAC3 is present in transcription corepressor complexes, we conditionally deleted Hdac3 in Tregs by crossing

$H d a c 3^{A / f l}$ and Foxp3-YFP/Cre mice. The resultant mice (hereafter, $\mathrm{HDAC3}^{-/-}$mice) lacked $\mathrm{Hdac} 3$ within their $\mathrm{FOXP3}^{+}$cells (Supplemental Figure 2). These mice proved sickly (Figure 2A) and died by 6 weeks of age unless WT Tregs were adoptively transferred at 2-3 days of life $(P<0.01)$ (Figure $2 \mathrm{~B})$. At 4 weeks of age, histologic examination of $\mathrm{HDAC}^{-/-}$mice showed dense mononuclear cell infiltration of lung (Figure 2C) and liver (Figure 2D) tissues, with only modest involvement of other organs (Supplemental Table 1). $\mathrm{HDAC}^{-/-}$mice also had markedly enlarged lymph nodes and spleens, atrophic thymuses (Figure 2E), and corresponding changes in total cellularity (Figure 2F). Hdac3 deletion in Tregs led to gross disruption of normal thymic $\mathrm{T}$ cell development, with markedly reduced overall cellularity and decreased double-positive and increased single positive thymocytes (Supplemental Figure 3), consistent with thymic destruction by autoreactive T cells (21).

Flow cytometric analysis showed that compared with WT controls, $\mathrm{CD}^{+}$and $\mathrm{CD}^{+} \mathrm{T}$ cells of $\mathrm{HDAC3}^{-/}$mice had increased expression of CD44 $4^{\mathrm{hi}}, \mathrm{CD} 62 \mathrm{~L}^{\mathrm{lo}}$, and CD69 activation markers and increased proliferation (Figure 3, A and B, and Supplemental Figure 4). As discussed below with regard to CCR7 and sphingosine-1phosphate receptor expression, $\mathrm{HDAC}^{-/-}$mice also had markedly decreased numbers of splenic but, surprisingly, increased lymph node and intrathymic Tregs (Figure 3, C and D).

Deletion of HDAC3 in FOXP3 ${ }^{+}$Tregs was accompanied by a significant increase in the proportions of activated $\mathrm{B}$ cells (Figure $3 \mathrm{E})$; increased production of $\operatorname{IgA}$, IgG1, IgG2a, IgG2b, IgG3, and IgM immunoglobulins (Figure 3F); and development of cryoglobulins (Supplemental Figure 5). Cryoglobulin formation was associated with the development of a mild proliferative glomerulonephritis (Supplemental Figure 6) - with glomerular deposition of IgM and C3, and neutrophil and macrophage infiltration - and development of mild proteinuria, though renal function remained 
A
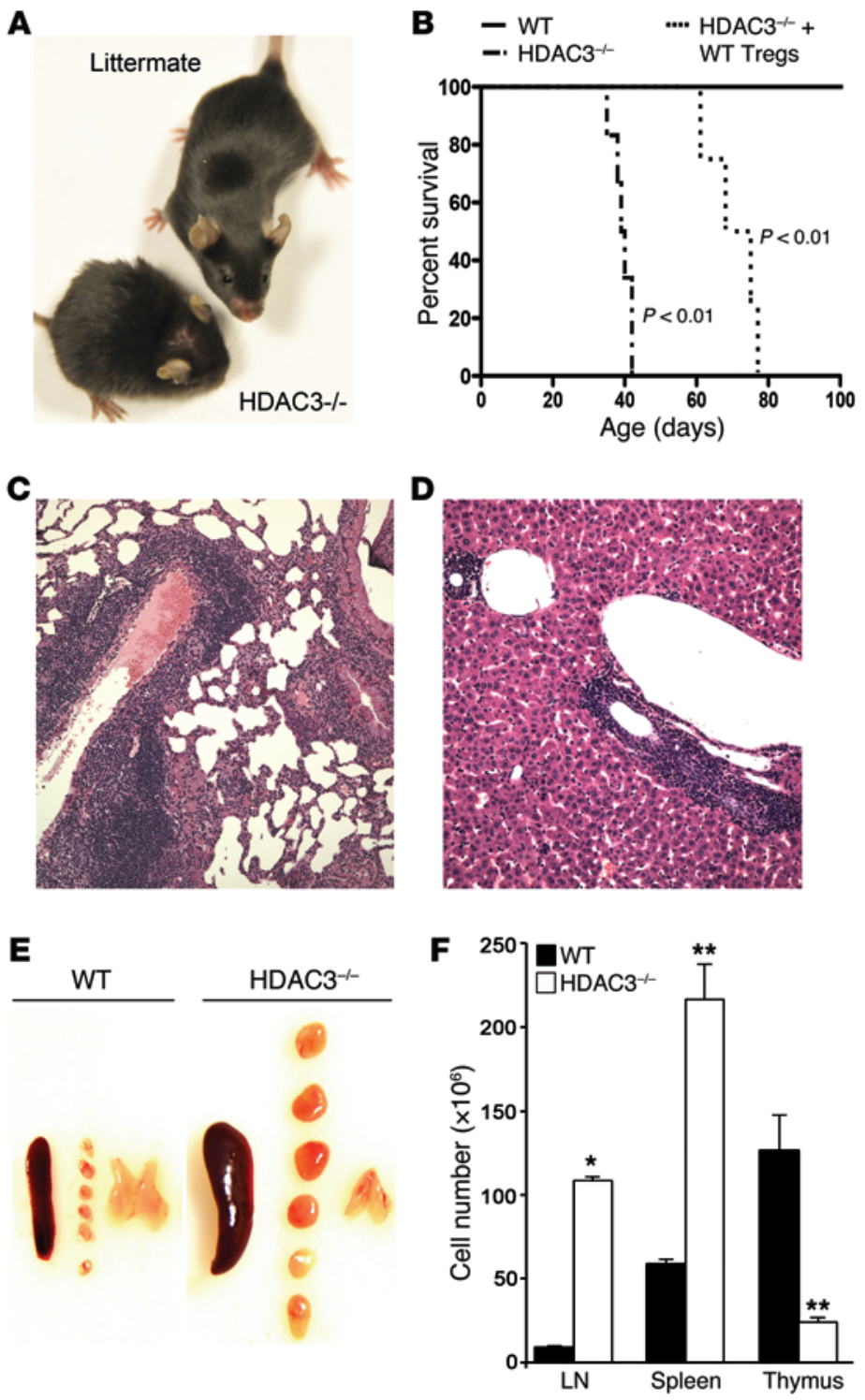

Figure 2. Hdac3 deletion in $\mathrm{FOXP3}^{+}$Tregs causes lethal autoimmunity. Data in all panels except $\mathbf{B}$ are from 6-8 mice/group studied at 4 weeks of age. (A) Typical littermate and $\mathrm{HDAC}^{-1-}$ mice. (B) Early death of $\mathrm{HDAC}^{-1-}$ mice unless they received adoptive transfer of $1 \times 10^{6} \mathrm{WT}$ Tregs (6-8 mice/group, Kaplan-Meier plots followed by log-rank test). (C) Lung infiltrates and (D) portal infiltrates in livers of $\mathrm{HDAC3}^{-1-}$ mice (original magnification, $\times 200$ ). (E) Comparison of spleen, lymph nodes, and thymus of WT and $\mathrm{HDAC}^{-1-}$ mice. (F) Cellularity of lymph nodes $(\mathrm{LN})$, spleen, and thymus of WT and $\mathrm{HDAC3}^{-1-}$ mice (mean $\pm \mathrm{SD}, 6-8$ mice/group, Student's $t$ test for unpaired data; ${ }^{*} P<0.05$, ${ }^{* *} P<0.01$ )

3, C and D), we compared the functions of Tregs isolated from each site. HDAC3 ${ }^{-/-}$Tregs from both lymph nodes (Figure 4C) and spleens (Figure 4D) showed marked impairment of Treg function when compared with corresponding WT Tregs. In further support of the importance of HDAC3 in Tregs, retroviral transduction of $\mathrm{HDAC3}^{-/-}$Tregs with HDAC3 significantly improved Treg suppressive function (Figure $4 \mathrm{E}$ ). These data were surprising in that Foxp 3 mRNA expression was normal in $\mathrm{HDAC3}^{--}$Tregs, and the mRNA expression of other Tregrelated genes was either increased (Ctla4, Tgfb, Il10, Ebi3) or similar (Gitr) when compared with that of WT Tregs (Supplemental Figure 8). However, in addition to aberrant IL-2 production as noted above, $\mathrm{HDAC}^{-/-}$Tregs showed increased Ifng mRNA production, consistent with derepression of genes atypical of this lineage.

HDAC3 exists in transcription corepressor complexes containing two homologous proteins, nuclear receptor corepressor (NCoR1) and silencing mediator for retinoid and thyroid receptors (SMRT, also known as NCoR2) (23-25), plus inositol tetraphosphate (IP4). The limited intrinsic enzymatic activity of HDAC3 is markedly enhanced by its binding to the deacetylase-activating domain (DAD) of NCoR1 or SMRT/ NCoR2 (19). IP4 helps stabilize this interaction and also triggers a conformational change in HDAC 3 that makes its catalytic site more accessible to substrate $(26,27)$. To test whether the interaction of HDAC3 with NCoR1 or SMRT/NCoR2 is required for optimal Treg function, we used mice with mutations of the DADs of both NCoR1 and SMRT/NCoR2 (28). These mice had normal proportions of FOXP3 ${ }^{+}$Tregs (Supplemental Figure 9) but decreased Treg suppressive function (Figure 4F), consistent with a role for NCoR1 or SMRT/NCoR2 in the regulation of HDAC3 function in Tregs. These findings indicate that HDAC3 and its interactions with NCoR1/SMRT are important for the ability of $\mathrm{FOXP}^{+}$Tregs to suppress $\mathrm{T}$ cell proliferation in vitro.

HDAC3 is essential for FOXP3 ${ }^{+}$Treg suppressive function in vitro. We used three animal models to assess the functions of HDAC3 $^{-/}$Tregs in vivo $(10,11,13)$. First, HDAC3 ${ }^{-/}$or WT Tregs plus conventional $\mathrm{T}$ cells were transferred to immunodeficient mice. Compared with WT Tregs, HDAC3 ${ }^{-1-}$ Tregs were very poor suppressors of the resultant homeostatic $\mathrm{T}$ cell proliferation (Figure $4 \mathrm{G}$ ). Second, we undertook cardiac allografting in immunodeficient hosts. Recipients adoptively transferred with conventional $\mathrm{T}$ cells developed acute rejection by 14 days after transplant, whereas mice receiving cotransfer of conventional $\mathrm{T}$ cells and WT Tregs (2:1 ratio) maintained the allografts long-term (>120 days). However, all grafts in mice receiving conventional vitro (Figure 4, A and B). As Treg numbers in $\mathrm{HDAC}^{-/-}$mice were
increased within lymph nodes but decreased in the spleen (Figure 
A

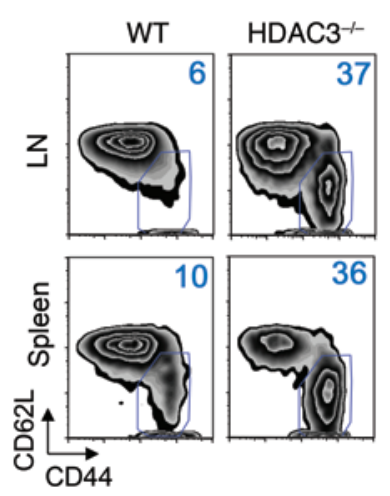

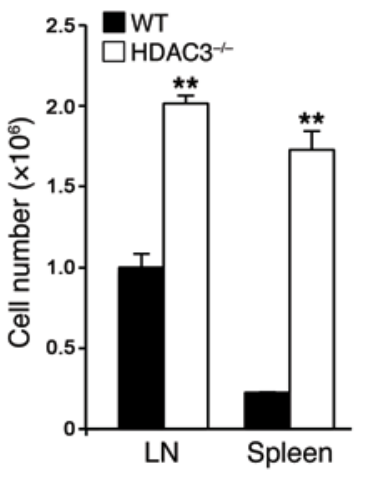

B

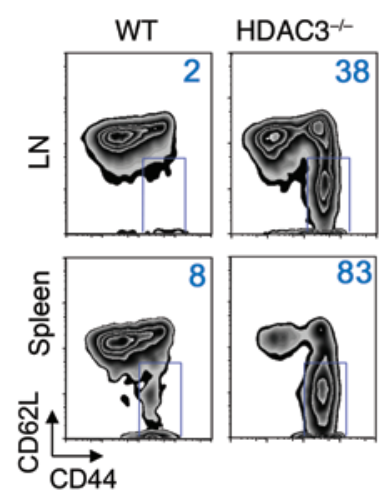

CD8 ${ }^{+} \mathrm{CD} 62 L^{10} \mathrm{CD} 44^{\text {hi }}$ cells

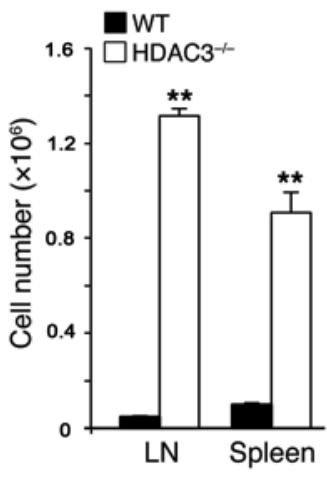

C

CD4+YFP+ cells
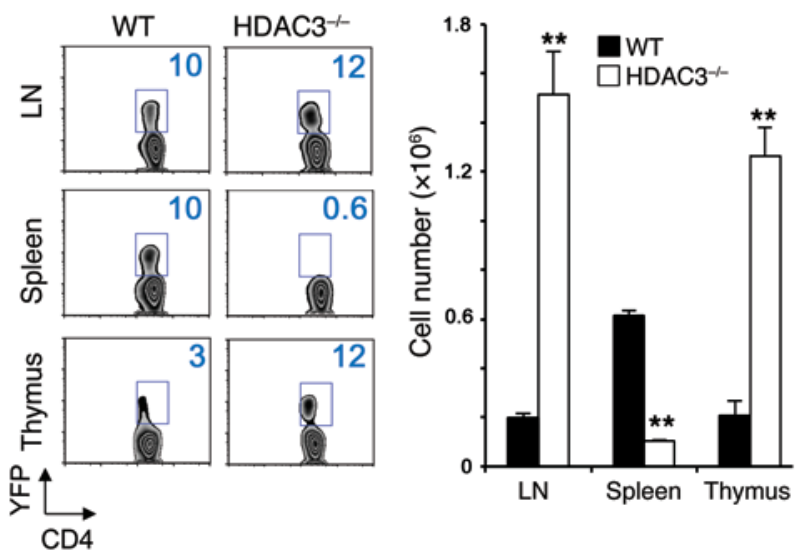

E

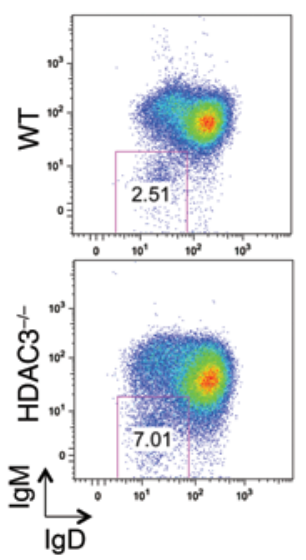

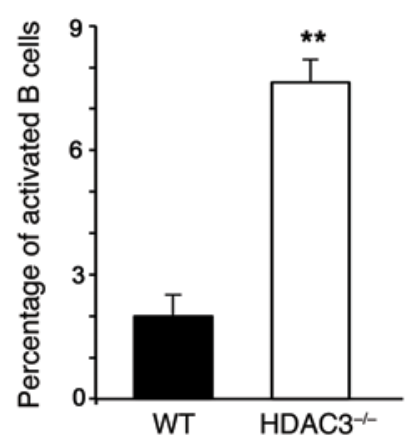

D

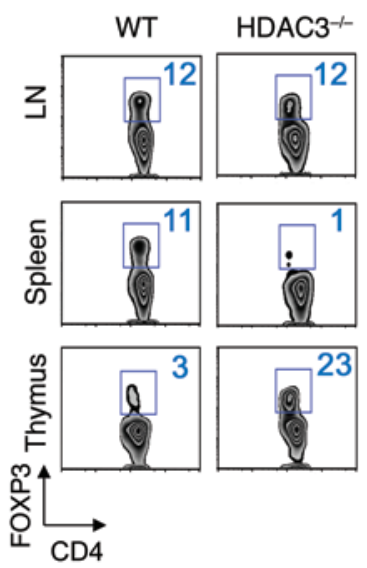

$\mathrm{CD}^{+}{ }^{\mathrm{FOXP}} 3^{+}$cells

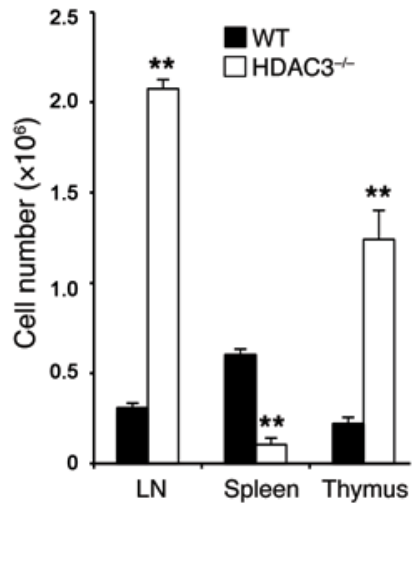

Figure 3. Hdac3 deletion in FOXP3+ Tregs causes activation of conventional T and B cells. Compared with WT controls, mice whose Tregs lack Hdac3 show expansion of $(\mathbf{A}) \mathrm{CD} 4{ }^{+} \mathrm{CD} 62 \mathrm{~L}^{\mathrm{l}} \mathrm{CD} 44^{\mathrm{hi}}$ cells and (B) CD8 ${ }^{+} \mathrm{CD} 6 \mathrm{~L}^{\mathrm{l}}{ }^{\circ} \mathrm{CD} 44^{\mathrm{hi}}$ cells in lymph nodes and spleens, with representative plots (left) and absolute numbers (right) in each panel; lymph node and thymic (C) YFP+ and (D) FOXP3 ${ }^{+}$cell populations, with representative plots (left) and absolute numbers (right) in each panel; (E) activated lymph node and splenic CD19+ B cells (IgM $\mathrm{I}^{10} \mathrm{~g} \mathrm{~g}^{10}$ ), with representative plots (left) and percentages (right); and (F) all main immunoglobulins. Data are shown as mean \pm SD, 6-8 mice/group, Student's $t$ test for unpaired data; ${ }^{*} P<0.05$ and ${ }^{*} P<0.01$ vs. WT control.

T cells and $\mathrm{HDAC}^{-/-}$Tregs were rejected by 33 days after transplant (Figure 4H). Third, we tested the effects of $H d a c 3$ deletion in an adoptive transfer model of colitis using a 1:2 ratio of Tregs to conventional T cells (13). Compared with use of WT Tregs, mice adoptively transferred with $\mathrm{HDAC}^{-/-}$Tregs showed increasing weight loss from 2 weeks after cell transfer (Figure 5A), and by sacrifice at 7 weeks after transfer, they had shortened, thickened colons (Figure 5B). Histology showed markedly increased mononuclear cell infiltration in colons from recipients of $\mathrm{Hdac}^{-/-}$Tregs, loss of goblet cells, transmural thickening, and increased $\mathrm{CD}^{+} \mathrm{T}$ 
A

WT
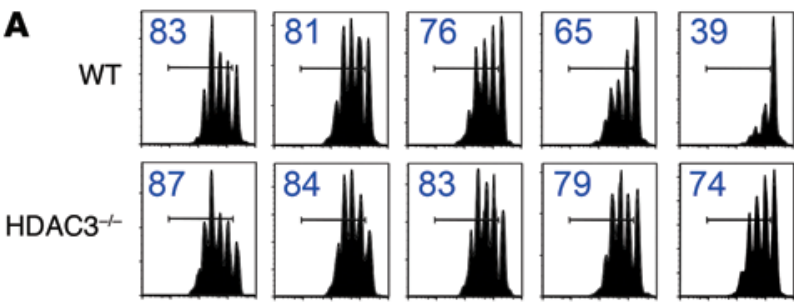

$0: 1$

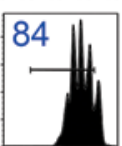

$1: 8$

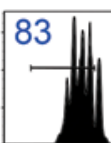

$1: 4$

Treg:Teff ratio

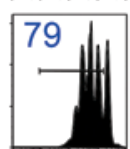

$1: 2$

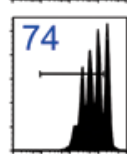

$1: 1$

C
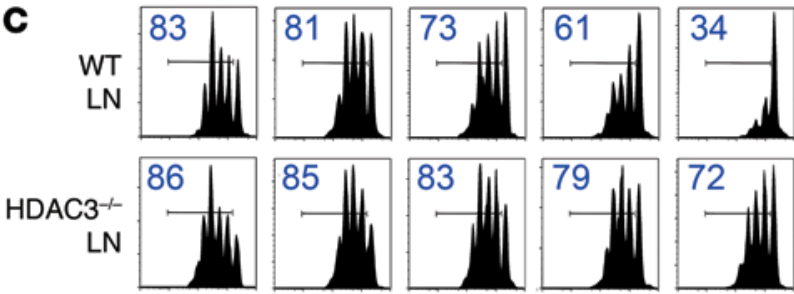

$0: 1$
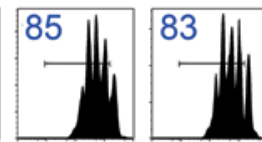

$1: 4$

Treg:Teff ratio

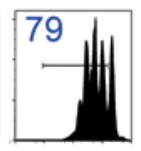

$1: 2 \quad 1: 1$

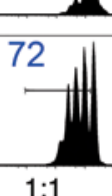

E

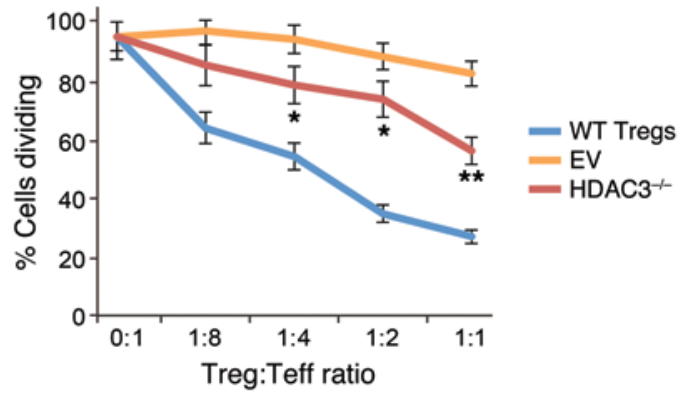

G

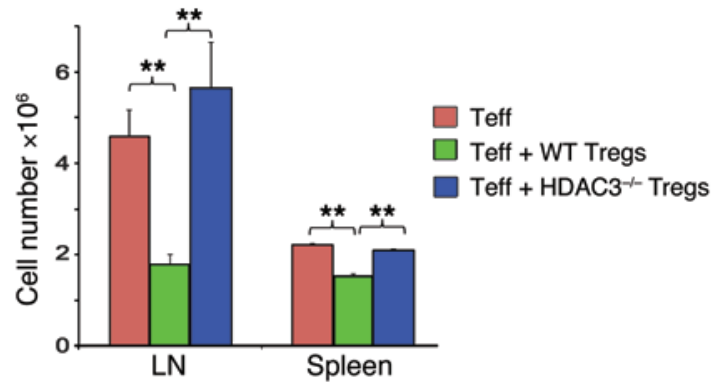

B

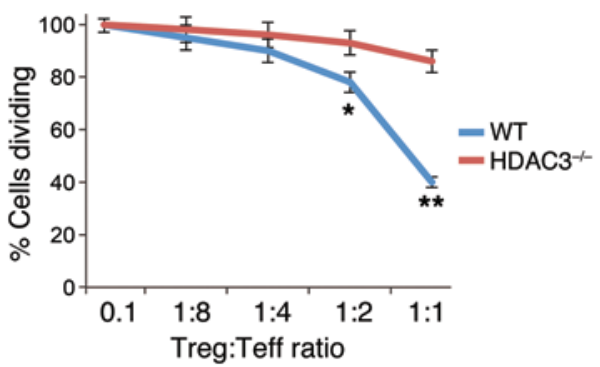

D
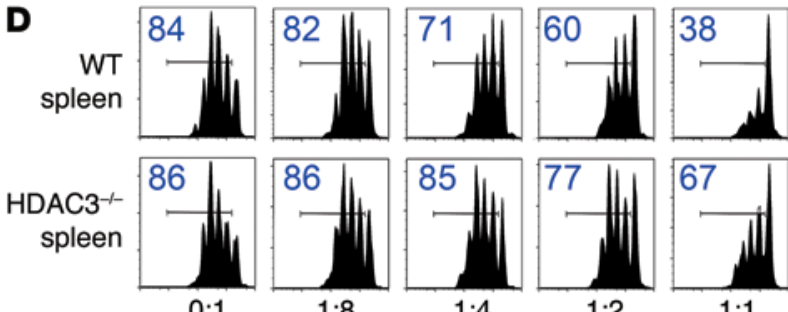

$1: 8$

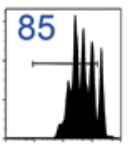

$1: 4$

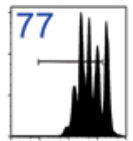

$1: 2$

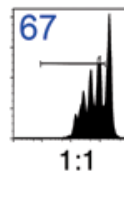

$\mathbf{F}$
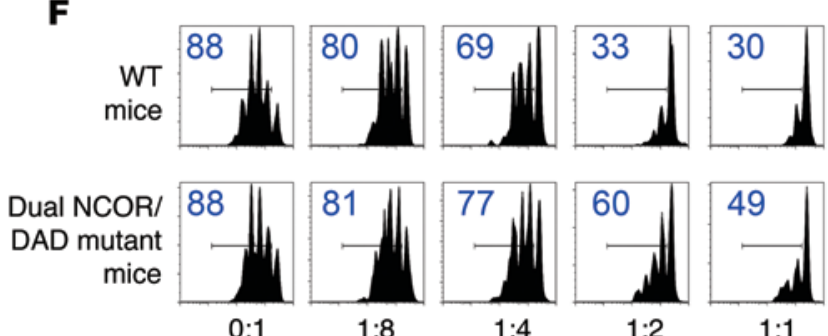

$1: 4$

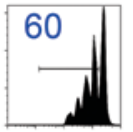

$1: 2$

Treg:Teff ratio

H

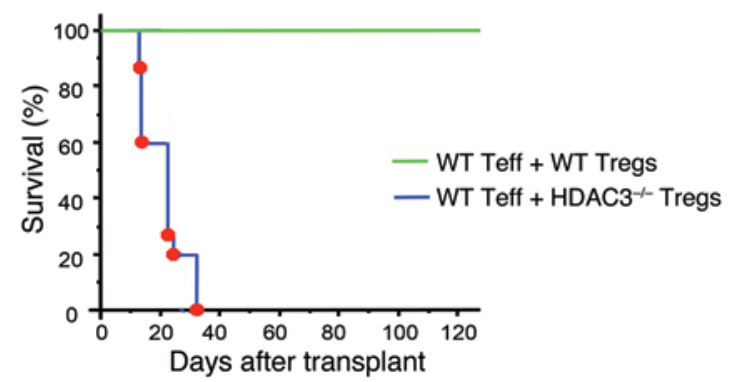

Figure 4. Hdac3 deletion impairs Treg function in vitro and in vivo. (A-F) In vitro experiments. (A) Treg suppression assay using pooled Tregs from lymph nodes and spleens of WT and HDAC $3^{-1-}$ mice, with representative data shown in $\mathbf{A}$, along with the percentage of proliferating cells in each panel; pooled data (4 mice/group) are shown in B. Corresponding in vitro Treg assays using purely (C) lymph node Tregs and (D) splenic Tregs. (E) Retroviral transduction of Hdac3 significantly improved $\mathrm{Hdac}^{-/-}$Treg function compared with empty vector transduction. (F) Treg suppression assay using Tregs from mice with mutations of DADs in both NCoR1 and SMRT/NCoR2. (G and H) In vivo experiments. (G) Contrasting effects of WT and Hdac $3^{-/-}$Tregs on the extent of homeostatic proliferation at 7 days after adoptive transfer of WT Teffs injected into Rag ${ }^{-/-}$mice. (H) Contrasting survival of BALB/c hearts transplanted into C57BL/6 Rag1/- mice and adoptively transferred with $1 \times 10^{6}$ C57BL/6 Teffs and $0.5 \times 10^{6}$ WT or Hdac3-/- C57BL/6 Tregs (Kaplan-Meier plots, 6 allografts/group). Each experiment in A-G was run in triplicate and repeated at least 3 times, and results of 1 representative experiment are shown; mean \pm SD, 1-way ANOVA with corresponding Tukey's multiple comparison test; ${ }^{*} P<0.05$ and ${ }^{*} P<0.01$ vs. WT control.

cells but fewer FOXP3 ${ }^{+}$Tregs (Figure 5C). Analysis confirmed a significant reduction in colon length (Figure 5D) and increased histologic scores of colitis (Figure $5 \mathrm{E}$ ) in the mice receiving $\mathrm{Hdac}^{-1-}$ versus WT Tregs. Last, flow cytometry showed increased numbers of conventional $\mathrm{YFP}^{-} \mathrm{T}$ cells in lymphoid tissues (Figure $5 \mathrm{~F}$ ) and reduced $\mathrm{YFP}^{+}$Tregs (Figure 5G) at 7 weeks after transfer of $\mathrm{Hdac}^{-/-}$versus WT Tregs. Hence, HDAC3 is important for normal Treg suppressive function in vivo, and in its absence host conventional $\mathrm{T}$ cells promote tissue injury.

Hdac3 deletion does not limit Treg proliferation or increase Treg apoptosis. We then considered whether the altered numbers and function of Tregs in $\mathrm{HDAC3}^{-/-}$mice reflected reduced proliferative capacity or increased apoptosis. While Tregs are normally quite proliferative in vivo, we found that BrdU incorporation was sig- 


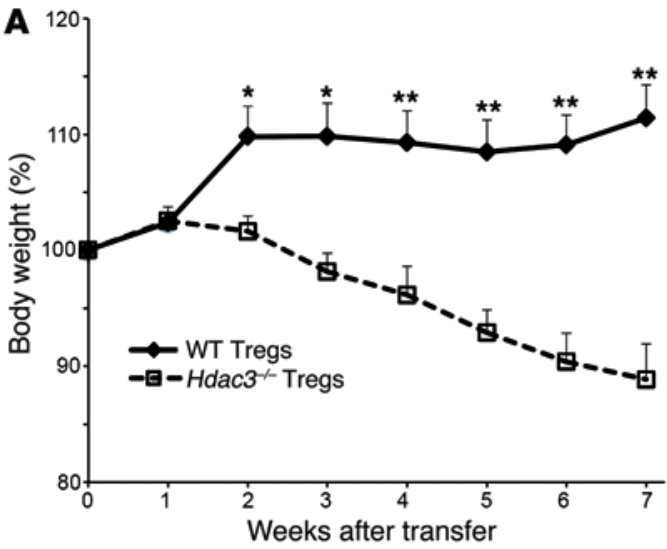

$\mathbf{B}$
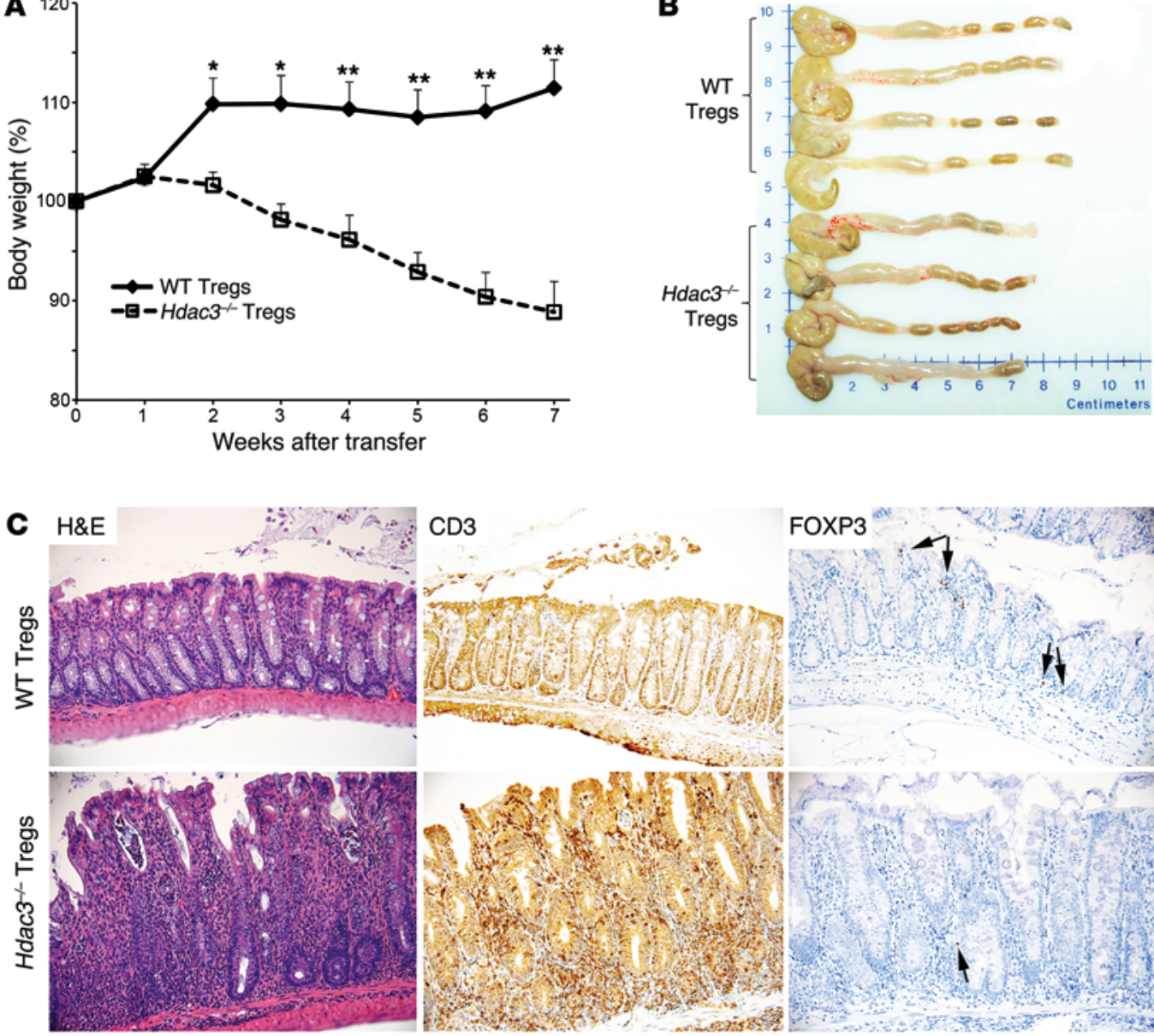

CD3

D

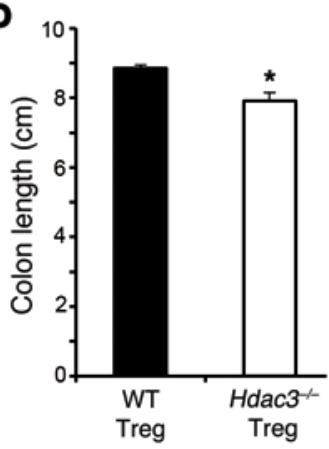

E

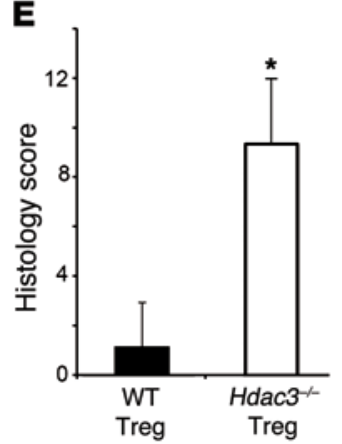

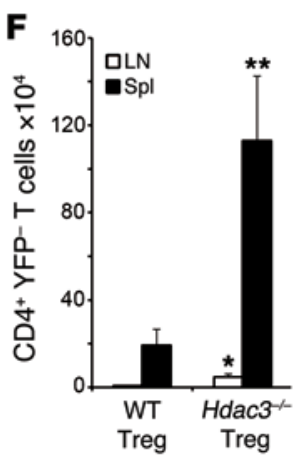

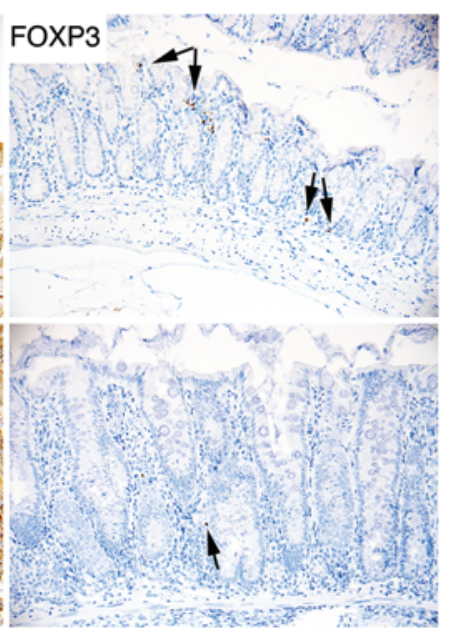

G

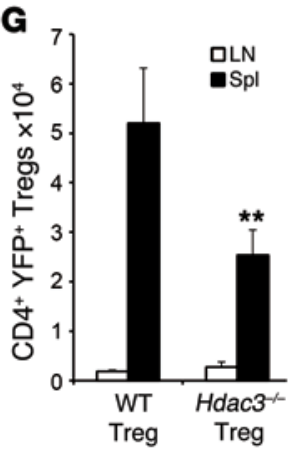

Figure 5. Hdac3 deletion impairs Treg function in an adoptive transfer model of colitis (2:1 ratio of Tcon cells to Tregs). (A) In contrast to WT Tregs, transfer of $\mathrm{Hdac}^{-1-}$ Tregs could not prevent the development of colitis. (B) Macroscopic appearance of colons from mice receiving WT or $\mathrm{Hdac}^{-/-}$Tregs. (C) Histologic comparisons (original magnification, $\times 200$ ) of colons in mice receiving WT or $\mathrm{Hdac}^{-1-}$ Tregs show that the latter have transmural thickening, loss of goblet cells and dense infiltrates, increased $\mathrm{CD}^{+}$cells, but a paucity of Tregs (arrows). Compared with mice receiving WT Tregs, mice receiving $\mathrm{Hdac}^{-/-}$Tregs had (D) shortened colons; (E) greater histologic scores of colitis; (F) higher numbers of conventional $T$ cells within lymph nodes and spleens (Spl), but (C) fewer $\mathrm{YFP}^{+} \mathrm{FOXP3}^{+}$Tregs at these sites. Studies involved 9 mice/group, mean \pm SD, with analysis by 1-way ANOVA with corresponding Tukey's multiple comparison test (A) or Student's $t$ test for unpaired data $(\mathbf{D}-\mathbf{C})$; ${ }^{*} P<0.05$, ${ }^{* *} P<0.01$ vs. WT control. nificantly greater in both Tregs and Teffs of $\mathrm{HDAC}^{-/-}$versus WT mice (Figure 6A). Moreover, compared with WT cells, Annexin V and 7-AAD staining of $\mathrm{HDAC}^{-/-}$Tregs and Teffs showed far greater percentages of Annexin $\mathrm{V}$ and 7-AAD double-negative cells (i.e., live cells) after $\mathrm{CD} 3 / \mathrm{CD} 28 \mathrm{mAb}$ activation in vitro (Figure 6B). HDAC3 ${ }^{-/-}$Tregs also had higher Bcl2 and lower Fasl gene expression than WT Tregs after activation (Figure 6C). Last, $\mathrm{HDAC3}^{-/-}$Thy $1.2^{+}$Tregs injected into Thy $1.1^{+}$mice were recovered in significantly higher numbers than WT Tregs after 4 weeks in vivo (Figure 6D). Hence, the decreased numbers and function of Tregs in $\mathrm{HDAC}^{-/-}$mice are unlikely to result from decreased Treg proliferation or increased Treg apoptosis.

HDAC3 expression is required for iTreg development. Extrathymic $\mathrm{FOXP}^{+}$Tregs comprise a mixture of thymus-derived or natural
Tregs (nTregs) and peripherally induced Tregs (iTregs). Thymic production of Tregs was unimpaired in $\mathrm{HDAC3}^{-/-}$mice (Figure 3), and $\mathrm{HDAC}^{-/-}$Tregs were not more prone to apoptosis (Figure 6). This led us to consider whether the drastic reduction in $\mathrm{HDAC}^{-/-}$ Tregs within the spleen, associated with extensive conventional $\mathrm{T}$ (Tcon) cell activation (Figure 3), might reflect impaired iTreg production. To test this, we crossed $\mathrm{Hdac} 3^{\mathrm{f} / \mathrm{fl}}$ and $\mathrm{Cd} 4$-Cre mice, isolated their $\mathrm{HDAC}^{-/-} \mathrm{CD} 4^{+} \mathrm{CD} 25^{-}$Tcon cells, and assessed their conversion into iTregs during 5 days of culture in vitro with CD3/ CD28 mAbs plus TGF- $\beta$ and IL-2 (10). We found that HDAC3deficient Tcon cells proliferated only slightly less than WT cells upon CD3 mAb stimulation (Supplemental Figure 10), but almost completely lost the ability to convert to iTregs under Treg polarizing conditions (Figure 7A), and showed significantly impaired 
A
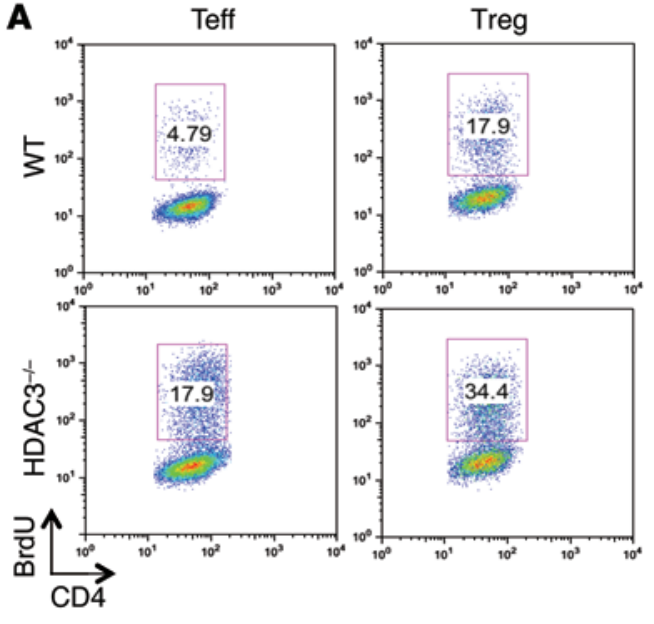

B

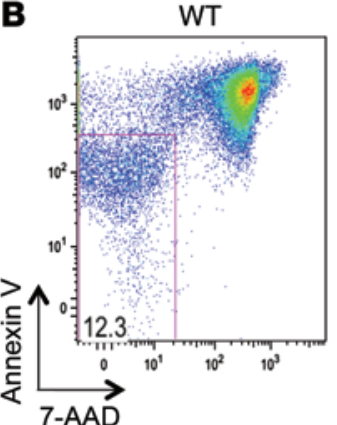

Treg

$\mathrm{HDAC}^{-1-}$

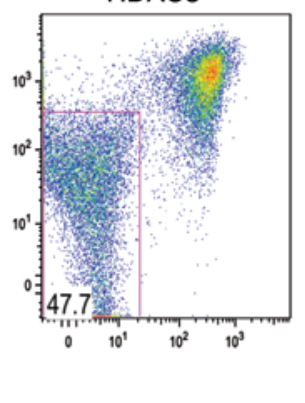

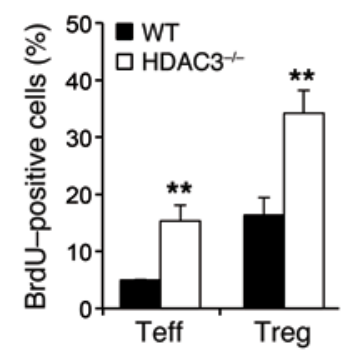

C

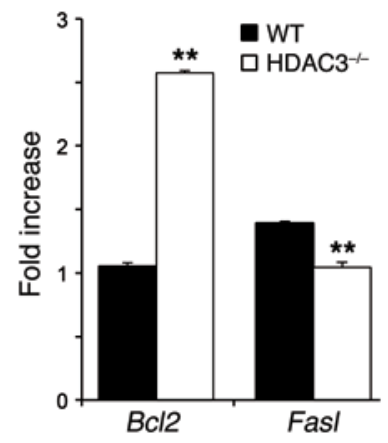

Figure 6. Hdac3 deletion does not affect Treg proliferation or apoptosis. (A) BrdU+ cells 3 days after injection of BrdU into WT or Hdac3 ${ }^{-/-}$mice, with percentages (left) and absolute numbers (right) of labeled cells. (B) Assessment of apoptosis after TCR stimulation (24 hours), with percentages (left) and absolute numbers (right) of double-negative (Annexin $\mathrm{V}^{-}, 7-\mathrm{AAD}^{-}$) viable cells. (C) Bc/2 and Fas/ mRNA expression in Tregs activated in vitro for 24 hours with CD3/CD28 beads (1:1 ratio, qPCR, 4/group). (D) Treg survival in vivo. $0.1 \times 10^{6}$ WT or Hdac3 ${ }^{-/-}$Tregs (Thy1.2+) isolated by cell sorting were injected into Thy $1.1^{+}$mice and numbers of Foxp3 ${ }^{+}$Thy $1.2^{+}$cells determined by flow cytometry at 1 week. Data are shown as mean \pm SD, 4-6 samples/group, Student's $t$ test for unpaired data; ${ }^{*} P<0.01$ and ${ }^{* *} P<0.001$ vs. WT control.

induction of Foxp3 gene expression (Figure 7B). Moreover, in contrast to WT cells treated in the same manner, HDAC3 $3^{-/-}$Tcon cells produced significantly increased amounts of IL-2 (Figure 7, C and D), IL-6 (Figure 7E), and IL-17 (Figure 7F).

TGF- $\beta$-induced iTreg generation in vitro begins with the binding of SMAD2 or SMAD3 to a conserved noncoding DNA sequence (CNS1) within the Foxp3 locus $(29,30)$, and in the absence of a normal CNS1 element, iTreg formation is greatly impaired (21). Using trimethylation of histone $\mathrm{H} 3$ at Lys9 (H3K9me3) as a marker of a closed, non-permissive chromatin structure, we undertook ChIP assays of $\mathrm{HDAC3}^{-/-}$and WT T cells cultured under the iTreg polarizing conditions noted above. We pulled down chromatin with an anti-H3K9me3 Ab and undertook analysis by qPCR of immunoprecipitated Foxp3 promoter and CNS1 and CNS2 elements. Compared with WT cells, HDAC3-deficient T cells showed increased H3K9me3 at the Foxp3 promoter and CNS2 regions, but there was no significant difference at the CNS1 site between the two groups (Figure 8A). CNS1 remodeling and accessibility are essential but not sufficient for iTreg generation, especially if the Foxp3 promoter and CNS2 sites remain inaccessible (21).

Consistent with data shown in Figure 6, we found greater acetylation of histone $\mathrm{H} 3$, indicative of increased accessibility at the $I l 2$ promoter of HDAC3-deficient versus WT T cells, and acetylation was further increased by $\mathrm{T}$ cell receptor (TCR) stimulation (Figure 8B). We also assessed chromatin modifications at the Il6 and Il17 promoter sites, since IL-6, in combination with TGF- $\beta$, is a potent driving factor for Th17 cell differentiation and IL-17 production. As considerable IL-6 mRNA and protein expression were observed in HDAC3-deficient CD4 ${ }^{+} \mathrm{T}$ cells under iTreg polarizing conditions (Figure 7), we also tested whether HDAC3 regulated IL-6 transcription. We found that HDAC3 normally binds to the Il6 promoter, and in the absence of HDAC3, levels of acetylated histone $\mathrm{H} 3$ at the $\mathrm{Il} 6$ promoter were significantly increased compared with WT controls (Figure 8C). In contrast, HDAC3 did not bind directly to the Il17 promoter (Figure 8D), but levels of acetylated histone $\mathrm{H} 3$ at this site were greatly increased in resting and activated HDAC3-deficient versus corresponding WT cells (Figure 8D). Collectively, these data indicate that while the CNS1 element is permissive for iTreg generation in $\mathrm{HDAC3}^{-/-}$cells, the absence of HDAC3 limits accessibility at the Foxp3 promoter and CNS2 sites and is accompanied by uncontrolled IL-2 and IL- 6 production that inhibits iTreg generation.

Profound effects of HDAC3 deletion on gene expression in Tregs. We undertook microarray analyses of $\mathrm{HDAC}^{-/-}$and WT Tregs to assess Hdac3-dependent global changes in gene expression. Functional annotation clustering showed enrichment in $\mathrm{HDAC3}^{-/-}$versus WT Tregs of genes that are associated with inflammatory and 

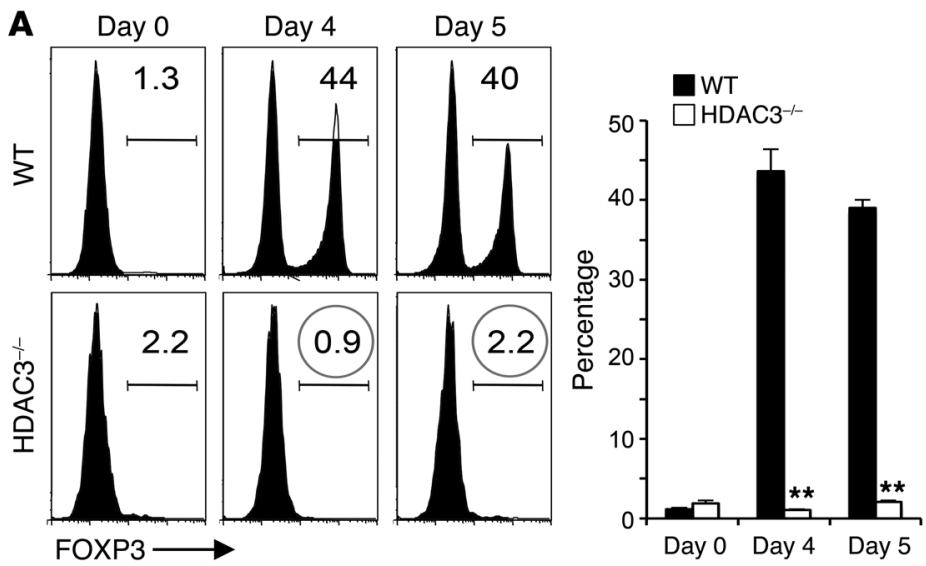

B
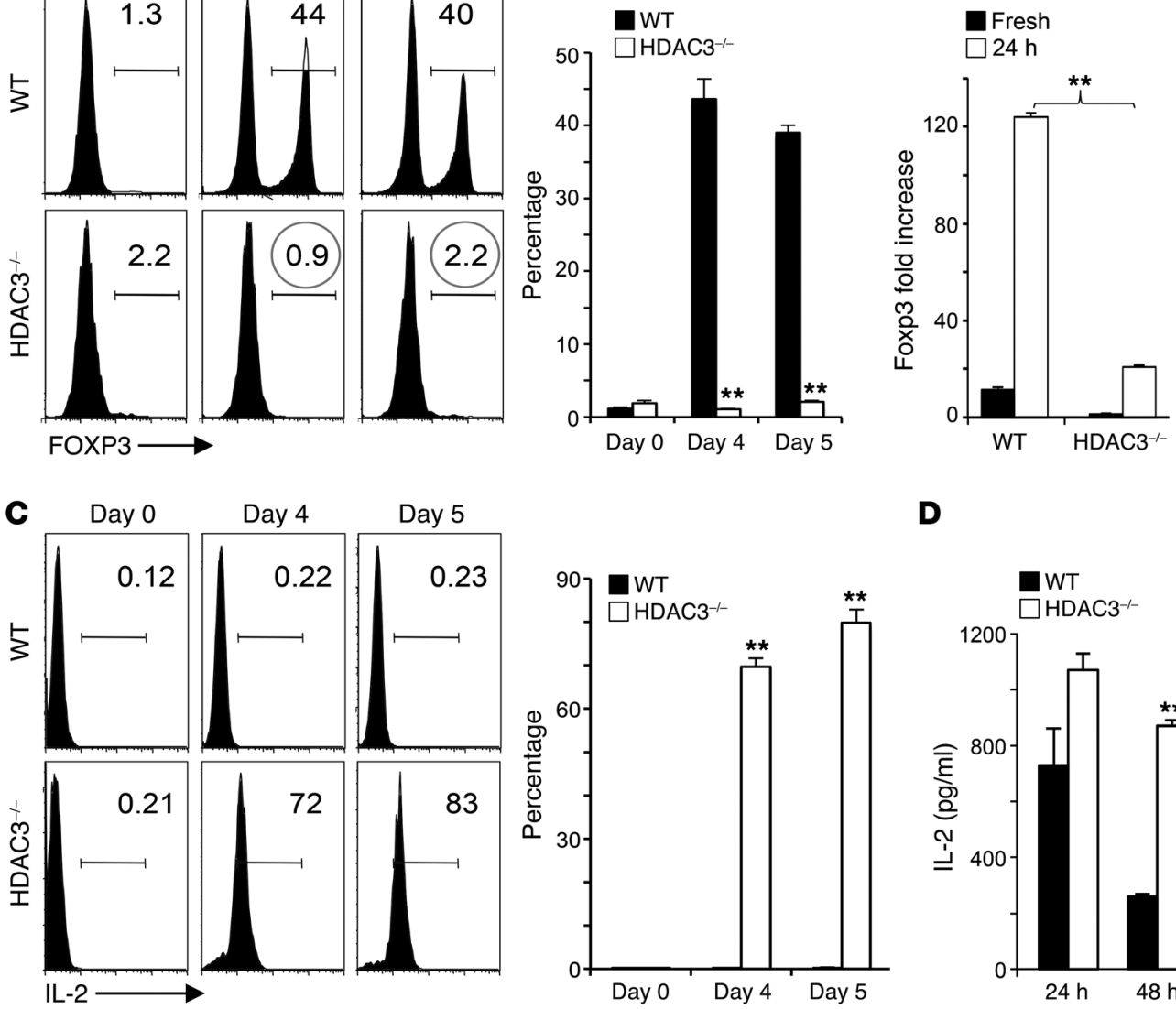

D
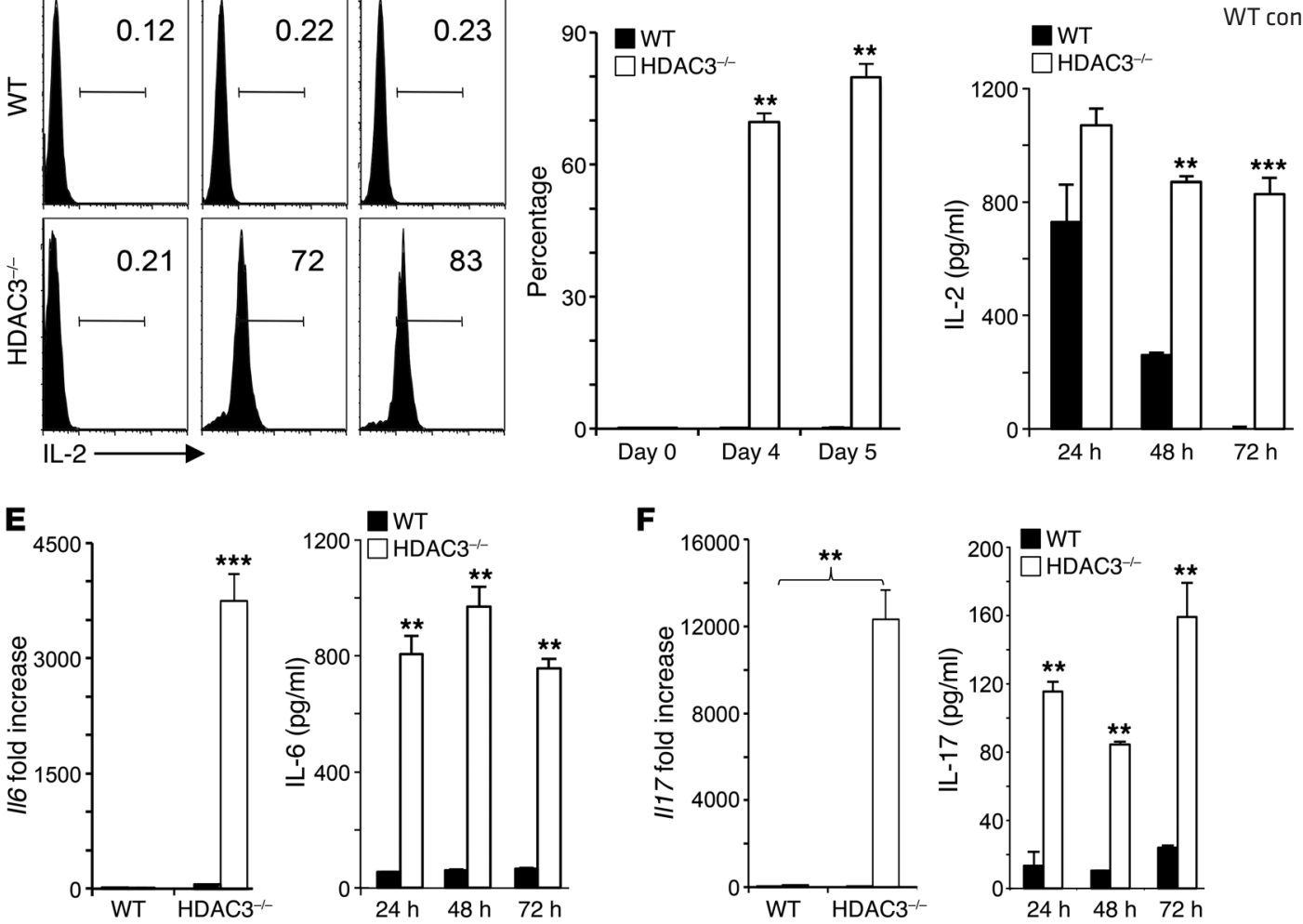

Figure 7. Hdac 3 is required for iTreg development. All panels represent conventional WT or $\mathrm{Hdac}^{-/-}$T cells cultured under Treg polarizing conditions and studied serially, as indicated. (A) Serial Foxp3 staining, with representative (left) and cumulative data (right) of $\mathrm{FOXP3}^{+}$cells. (B) Foxp3 mRNA expression after 24 hours polarization. (C) Serial IL-2 staining, with representative (left) and cumulative data (right) of IL-2+ cells. (D) Serial ELISA measurement of IL-2 protein levels. (E) IL-6 gene expression (left) and protein levels (ELISA) (right). (F) IL-17 gene expression (left) and protein levels (ELISA). Data are shown as mean $\pm S D$, 4-6 samples/ group, Student's $t$ test for unpaired data; ${ }^{* *} P<0.01$ and ${ }^{* *} P<0.001$ vs. WT control. immune responses, and decreased expression of genes associated with protein transcription and translation (Figure 9A). HDAC3 is reported to deacetylate p65 (RelA), a key member of the NF- $\mathrm{B}$ family $(31,32)$. Once deacetylated, p65 disassociates from DNA and is exported from the nucleus to the cytoplasm, but in the absence of HDAC3, p65 remains acetylated within the nucleus and induces ongoing gene transcription. Without TCR stimulation, p65 bound to I $\kappa \mathrm{B} \alpha$ and remained in the cytoplasm in WT Tregs (Figure 9B), whereas, even in resting Tregs, p65 was mainly located within nuclei of $\mathrm{HDAC3}^{-/-}$Tregs. Such ongoing activation of NF- $\kappa \mathrm{B}$ target genes likely contributes to the broad increase in production of $I l 2$, Il6, and additional proinflammatory cytokines (Figure 9C), chemokines (Figure 9D), and chemokine and adhesion-related receptors (Figure 9E and Supplemental Figure 11) in $\mathrm{HDAC3}^{-/-}$versus WT Tregs. Conditional deletion of $\mathrm{Hdac} 3$ in mice has been reported for hepatocytes $(33,34)$, cardiac and/or skeletal muscle cells $(35,36)$, osteo-chondroprogenitor cells (37), hippocampal cells (38), macrophages (39), T cells (40), and hemopoietic progenitor cells (41). Collectively, loss of $\mathrm{Hdac} 3$ caused cell type-specific changes in gene expression, including expression of imprinted genes, cell-cycle control, and growth factor/receptor signal transduction genes. While deletion of Hdac3 in Tregs led to increased expression of genes that were unaffected when HDAC3 was deleted in other cell types (Figure 9F), for the most part there were similar changes in gene expression (Figure 9G), as was reported for Hdac3 deletion in nonlymphoid cells $(33-39,41)$. Overall, Hdac3 deletion led to altered expression of a wide variety of genes in Tregs, including those promoting activation of NF- $\mathrm{BB}$ and other proinflammatory pathways.

\section{Discussion}

Conceptually, there are two broad strategies to harness Tregs for therapeutic purposes, namely transfer of expanded numbers of 
A

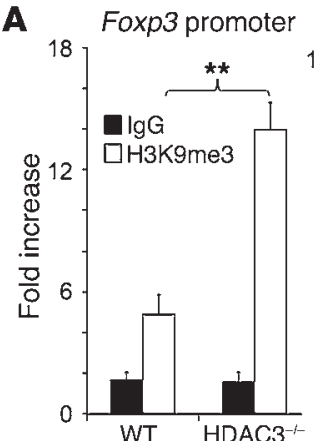

Foxp3 CNS1

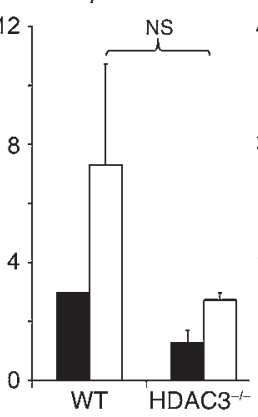

Foxp3 CNS2

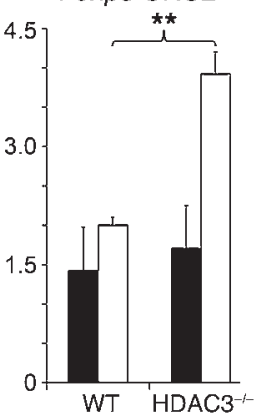

B

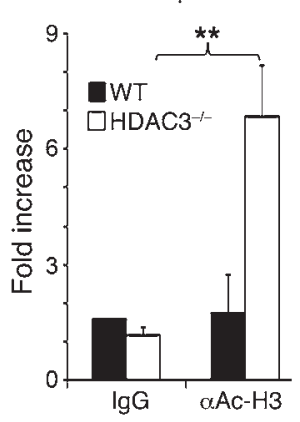

$24 \mathrm{~h} / 12$ promoter

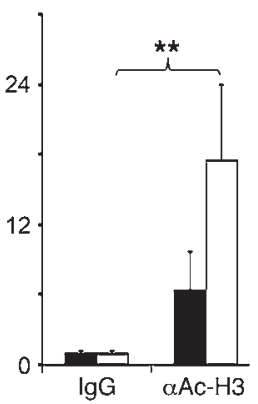

C

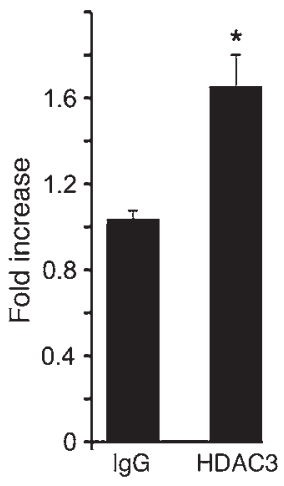

116 promoter

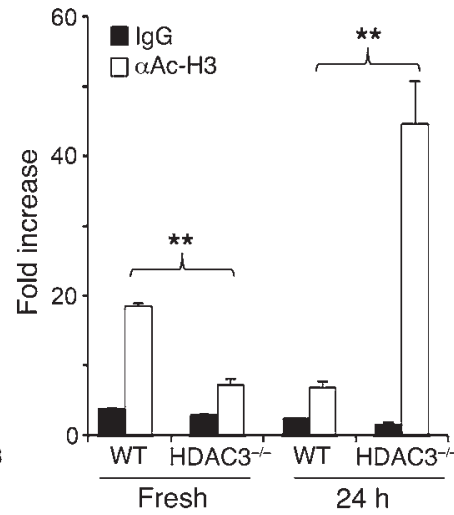

D

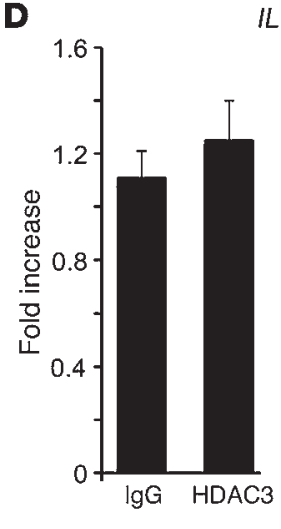

IL17 promoter

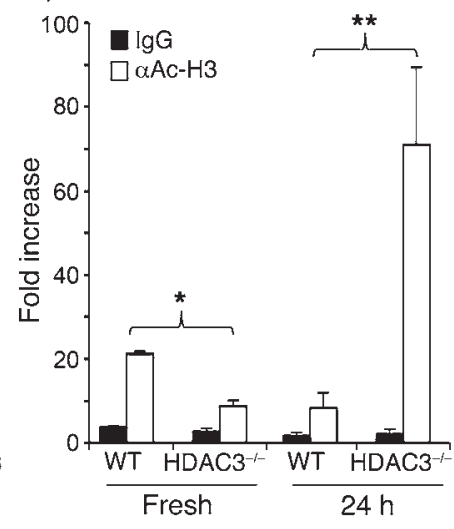

Figure 8. Hdac3 expression affects chromatin remodeling at Foxp3, II2, II6, and II17 promoters of T cells cultured under iTreg polarizing conditions. (A) ChIP assay of Foxp3 promoter (left) and CNS1 (middle) and CNS2 (right) with anti-histone H3K9me3 antibody pull-down. (B) ChIP analysis of acetylated histone $\mathrm{H} 3(\alpha \mathrm{AC}-\mathrm{H} 3)$ at the $/ 12$ promoter (left, unstimulated and right, after 24 hours polarization). (C) ChIP detection of $/ / 6$ promoter with anti-HDAC3 antibody pull-down (left) and ChIP for acetylated histone $\mathrm{H} 3$ level at the II6 promoter (right). (D) ChIP for the II17 promoter with anti-HDAC3 antibody pulldown (left) and ChIP for acetylated histone H3 at the I/17 promoter. Data are shown as mean \pm SD, 4-6 samples/group, Student's $t$ test for unpaired data; ${ }^{*} P<0.05$ and ${ }^{* *} P<0.01$ vs. WT control.

autologous Tregs (5) and pharmacologic modulation of Treg production and function, e.g., through the actions of HDAC inhibitors (12). Much of the data pointing to HDACs as suitable targets for pharmacologic modulation of Tregs involve members of the relatively obscure class IIa HDAC family of enzymes (12). Class IIa HDACs each have a histidine rather than a tyrosine residue at a key site in their catalytic domains, which decreases catalytic activity by 1,000-fold compared with standard class I HDAC enzymes assayed using canonical substrates (15), such that they may better be described as pseudoenzymes. A search for noncanonical substrates of these proteins continues, with isolated reports of distinct catalytic activity $(42,43)$, as well as sites adjacent to the catalytic pocket potentially suitable for small molecule targeting (44). However, many now believe that class IIa HDACs function primarily as bromodomains (45-47). A practical extension of this point is that residues in the catalytic domain regions of class IIa HDACs can complex with class I HDACs, especially HDAC3 $(16,18)$. As a result, the HDAC3/NCoR complex may exert the deacetylase activity and suppressive actions originally ascribed to class IIa HDAC enzymes (16-19). The lack of potent and selective small molecule inhibitors of class IIa HDAC enzymes, and the association of class IIa HDACs with HDAC3, led us to determine the effects of HDAC3 deletion on FOXP3 ${ }^{+}$ Treg production and functions.
The lethal autoimmunity induced by Treg-specific deletion of $\mathrm{Hdac} 3$ was unexpected. While global deletion of Hdac3 during gestation is known to cause lethality by E9.5 (35), during the course of our work, CD4-Cre-mediated deletion of Hdac3 in all T cells was reported (40). In this study, the group did not find any alterations in conventional $\mathrm{T}$ cell development in their CD4-Cre Hdac3 conditionally deleted mice, though the mice failed to generate invariant NKT (iNKT) cells. In contrast, the current studies indicate that $\mathrm{Hdac} 3$ has unique functions in Tregs, and its loss cannot be compensated by other HDAC family members. Loss of Hdac3 leads to upregulation of a large number of inflammatory and immunerelated genes (Figure 9A), as well as persistent NF- $\mathrm{KB}$ activation (Figure 9B). The range of genes whose expression was altered, including many also upregulated when Hdac3 was deleted in other cell types (Figure 9G), points to a general repressive role of HDAC3 on gene expression in Tregs rather than one necessarily dependent on the functions of FOXP3. However, our data indicate that FOXP3dependent recruitment of HDAC3 contributes significantly to the repression of IL-2 production in Tregs and is also central to the development of iTregs. Loss of Hdac3 was accompanied by marked impairment of Treg suppressive function (Figure 4), with consequent activation of immune cells and their infiltration and damage of host tissues (Figures 2 and 3, and Supplemental Table 1). Hence, the deletion of Hdac3 has important FOXP3-dependent 
A

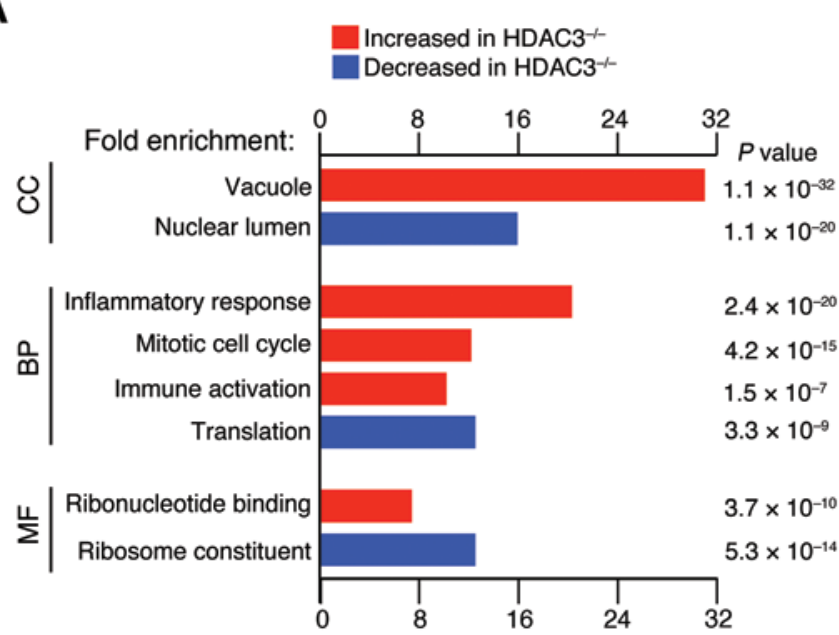

B
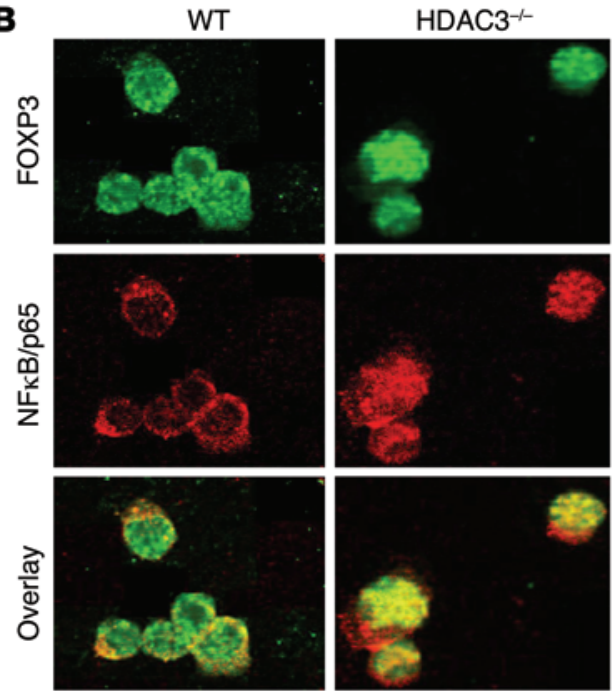

$\mathrm{HDAC}^{-1-}$
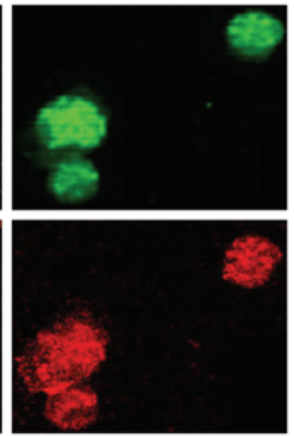

C

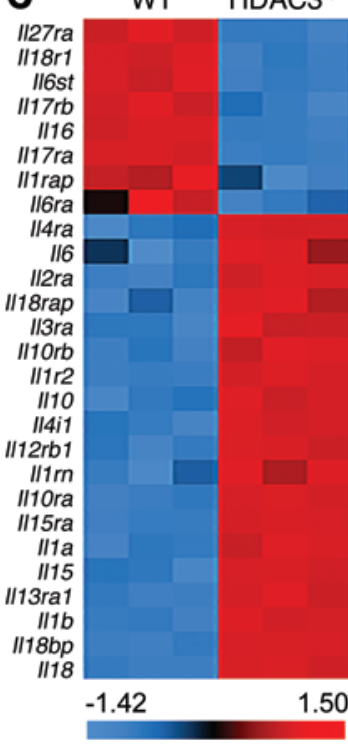

D

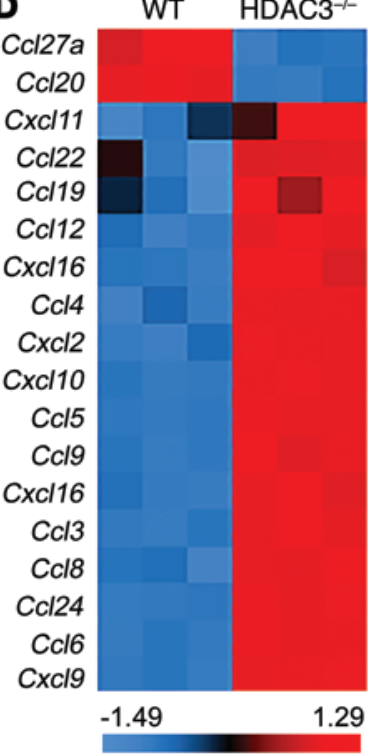

E

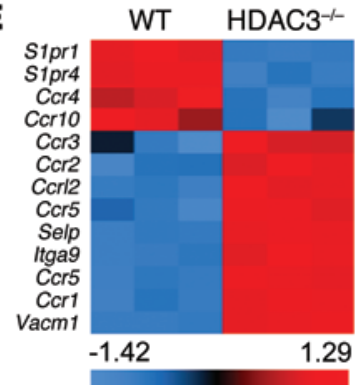

$\mathbf{F}$

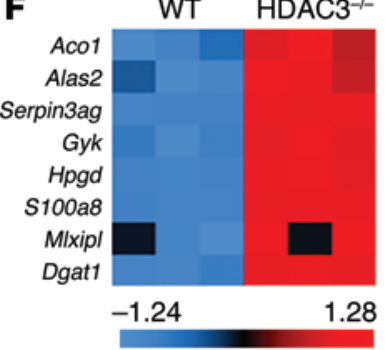

G

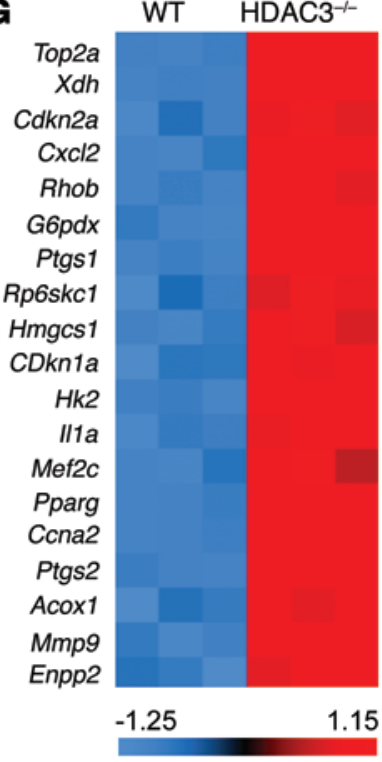

Figure 9. Effects of Hdac3 deletion on gene expression in FOXP3+ Tregs. (A) Functional annotation of microarray data. CC, cellular component; BP, biological process; MF, molecular function. (B) Immunofluorescence localization of NF- $\mathrm{kB} / \mathrm{p} 65$ and FOXP3 (original magnification, $\times 200$, representative of 3 independent experiments). Heat maps of microarray data associated with (C) cytokines and cytokine receptors, (D) chemokines, (E) chemokine receptors and adhesion molecules, and genes altered in Tregs (F) but not in other cell types when Hdac3 was deleted, or (G) genes also altered in other cell types when Hdac3 was deleted.

and FOXP3-independent effects on Treg development and function. As a result of these integral and complex roles that HDAC3 plays in Treg biology, we believe that pharmacologic approaches to enhance Treg function through modulation of HDAC3 need to be approached carefully, since they may lead to a critical impairment of host Treg functions.

Several additional points arising from our data are worth noting. First, Treg-dependent control of $\mathrm{T}$ cell help to B cells is a multifaceted and currently little-understood area. While Treg depletion is known to promote autoantibody production in murine models of arthritis and lupus, the effects of targeting individual proteins expressed by Tregs, especially beyond known functionally active surface molecules such as CTLA4, PD-1, and ICOS, are largely unexplored (48). We have recently shown that FOXP3dependent deletion of a DNA methyltransferase, DNMT1 (22), or a histone acetyltransferase, p300 (11), results in a distinct pattern of autoantibody production. The current study shows yet another variation on this theme, with production of cryoglobulins rather than any organ-specific or systemic autoantibodies. While the details of how Hdac3 deletion in Tregs can lead to cryoglobulin formation, immune complex glomerulonephritis, and proteinuria require elucidation, these data indicate that Tregs can influence $B$ cell function and autoantibody production in diverse ways.

Second, we were surprised by the accumulation of $\mathrm{HDAC3}^{-/-}$ $\mathrm{FOXP}^{+}$Tregs in lymph nodes and thymus versus spleen (Figure 3). Increased thymic Tregs may suggest an attempted compensatory 
host response to produce more Tregs given their impaired function in the periphery, through as-yet-unknown mechanisms, but this would not explain the difference in lymph node versus spleen data. However, the entry of Tregs across high endothelial venules into lymph nodes, but not the spleen, is critically CCR7 dependent (49), and CCR7 expression was upregulated in HDAC3 $3^{-/-}$Tregs (Supplemental Figure 10). Indeed, the expression of an additional 13 chemokine and homing receptors, and 18 chemokines, was upor downregulated by the absence of Hdac3. Two G protein-coupled receptors from the sphingosine-1-phosphate receptor family, S1pr1 and S1pr4, were downregulated, and these are needed for the egress of immune cells, including Tregs, from the thymus and lymph nodes via lymphatic vessels (50). This combination of increased CCR7 expression and decreased S1pr1 and S1pr4 expression likely results in a trapping of $\mathrm{HDAC}^{-/-}$Tregs in lymph nodes.

Third, recent studies using deacetylase-dead HDAC3 mutants, including mutations that interfere with DAD or IP4 binding, or substitutions of key HDAC3 catalytic residues, indicate important deacetylase-independent functions of HDAC3 in liver cells (51). While conventional small molecule HDAC inhibitors act to inhibit catalytic activity, it is conceivable that small molecule inhibitors may be developed to block the interaction of HDAC3 with the DAD regions of NCoR1 and/or SMRT/NCoR2 or the interaction of HDAC3 with IP4. The current studies show that DAD-dependent interactions with NCoR and/or SMRT/NCoR2 are important for HDAC3-dependent Treg suppressive function. Mice with point mutations in the DADs of NCoR1 and SMRT/NCoR2 have negligible HDAC3 activity, despite having normal levels of this deacetylase (28). Hence, our data point to a deacetylase-dependent role for HDAC3 in Tregs. Further work will be required to assess the relative contributions of HDAC3 deacetylase-dependent and -independent activities in Tregs. The latter deacetylase-independent functions may include NCoR1- or SMRT/NCoR2-mediated recruitment and stabilization of complexes regulating transcription in Tregs.

Last, these studies contribute to our developing knowledge of the roles of HDACs in $\mathrm{FOXP3}^{+}$Tregs. Interactions of the N-terminal domain of FOXP3 with class IIa HDACs such as HDAC7 and HDAC9 constrain FOXP3 interactions and Treg function $(10,52)$, whereas our current data indicate an important role for HDAC3 in maintenance of Treg functions. Such contrasting effects suggest the importance of understanding the roles of the various HDACs in Treg biology, and ultimately finding isoform-specific HDACi small molecules suitable for their targeting, so as to pharmacologically regulate Treg function in vivo in a safe and rational manner.

\section{Methods}

Mice. We used male WT BALB/c and WT and Rag1 ${ }^{-/-}$C57BL/6 mice from The Jackson Laboratory, plus previously described CD4-Cre (53), Foxp3-YFP/Cre (54), Hdac3/ff/ (55), and dual mutant NCoR/SMRT (28) mice. All mice were backcrossed on the C57BL/6 background at least 8 times and used at 6-8 weeks of age unless otherwise specified.

Antibodies and flow cytometry. Single-cell suspensions were stained with mAbs, obtained from BD Biosciences unless otherwise specified, that were directed against CD4 (Pacific blue, clone RM4-5, \#558107), CD8 (PE-Cy7, eBioscience, clone 53-6.7, \#25-0081), FOXP3 (PE-Cy5, eBioscience, clone FJK-16s, \#15-5773), CD62L (APC-Cy7, clone MEL-14, \#560514), IL-2 (PE, clone JES6-5H4, \#554428), IFN- $\gamma$
(Alexa Flour 647, clone XMG1.2, \#557735), CD44 (PE, clone IM7, \#553134), and CD25 (APC, eBioscience, clone PC61.5, \#17-0251) and acquired with a CyAn flow cytometer (Dako). We also purchased unconjugated CD3 (clone 145-2C11, \#553057) and CD28 (clone 37.51, \#553294) mAbs from BD.

Treg suppression assays. For in vitro studies, $5 \times 10^{4}$ cell-sorted $\mathrm{CD}^{+}{ }^{+} \mathrm{FP}^{+}$Tregs or bead-isolated $\mathrm{CD} 4{ }^{+} \mathrm{CD} 25^{+}$Tregs from Cd4-Cre mice were added to 96-well plates and serially diluted in medium (RPMI-1640 and 10\% FBS, plus penicillin/streptomycin). Equal numbers of CFSE-labeled $\mathrm{CD} 4^{+} \mathrm{CD} 25^{-} \mathrm{T}$ cells and $\gamma$-irradiated antigen-presenting cells (APCs) were then added, plus CD3 mAb (1 $\mu \mathrm{g}$ / $\mathrm{ml}$ ), and cultured for 3 days. Thereafter, cells were stained with CD4 $\mathrm{mAb}$ (Pacific blue); CFSE- and CD4-positive T cell proliferation was determined, and data were analyzed with FlowJo. For in vivo Treg suppression assays, $1 \times 10^{6} \mathrm{CD} 4^{+} \mathrm{CD} 25^{-}$Thy $1.1^{+}$and $0.5 \times 10^{6}$ Tregs were injected via the tail vein into $\mathrm{Rag1}^{-{ }^{--}}$mice. A week later, lymph node and spleens were harvested and stained with Thy1.1-PE and CD4Pacific blue, and the numbers of Thy $1.1^{+} \mathrm{T}$ cell cells were determined using a CyAn flow cytometer.

Treg conversion from Tcon cells. Magnetic beads (Miltenyi) isolated from $\mathrm{CD} 4^{+} \mathrm{CD} 25^{-}$Tcon cells were stimulated with $\mathrm{CD} 3 / \mathrm{CD} 28 \mathrm{mAb}-$ coated beads (1:1 ratio) in the presence of TGF- $\beta$ ( 2 nM) and IL-2 (10 U/ $\mathrm{ml}$ ). On days specified, cells were harvested, stained for CD4 (Pacific blue) and FOXP3 (PE-Cy5), and analyzed by flow cytometry (CyAn).

Hematology and autoantibody detection. Citrated blood samples were assessed using an automated hematology analyzer modified and calibrated for murine samples. Sera of 3-to 4-week-old WT or HDAC3-/mice were serially diluted and assessed for autoantibody production by indirect immunofluorescence staining of tissues from normal C57BL/6 mice; negative controls included use of pooled healthy sera and use of conjugated antibody alone, as described previously $(11,22)$.

Luminex. Serum immunoglobulin isotypes were assessed with a Multiplex mouse immunoglobulin assay kit (Millipore) using LiquiChip Luminex 100 (QIAGEN) according to the manufacturer's instructions (Luminex xMAP Technologies).

Cardiac transplantation. BALB/c hearts were harvested and transplanted into $\mathrm{Rag1}^{-/-}$(C57BL/6 background) recipients, as described previously (56). Tregs and Teffs were isolated using magnetic beads, and $1 \times 10^{6}$ Teff and a $0.5 \times 10^{6} \mathrm{WT}$ or $\mathrm{Hdac}^{-/-}$Tregs were injected i.v. into Rag1 $^{-1}$ mice $(\mathrm{C} 57 \mathrm{BL} / 6)$ that had received BALB/c cardiac allografts. Graft survival was monitored as a function of the ability of injected Tregs to suppress Teff cell-dependent alloreactivity and cardiac allograft rejection.

Adoptive transfer model of colitis. WT or $\mathrm{Hdac}^{-/-} \mathrm{CD} 4^{+} \mathrm{Foxp}^{+}\left(\mathrm{YFP}^{+}\right)$ Tregs and Foxp3- WT T cells isolated by cell sorting (BD Aria) were injected i.v. into Rag1 $^{-/-}$mice at a 1:2 ratio $\left(0.25 \times 10^{6}\right.$ Tregs: $0.5 \times 10^{6}$ conventional T cells, 9 mice/group). Weights were measured weekly, and at cessation, colons were harvested for measurement and histology and numbers of lymph node and splenic Tregs and conventional $\mathrm{T}$ cells determined by flow cytometry (CyAn). H\&E-stained, paraffin-embedded sections were scored by a pathologist blinded to treatment conditions using a scoring system (57) involving 8 parameters: (a) degree of lamina propria inflammation graded 0-3; (b) degree of mucin depletion evidenced by loss of goblet cells graded $0-2$; (c) reactive epithelial changes (nuclear hyperchromatism, random nuclear atypia, increased mitotic activity) graded 0-3; (d) number of intraepithelial lymphocytes per high-power field within crypts graded 0-3; (e) degree of crypt 
architectural distortion graded 0-3; (f) degree of inflammatory activity (infiltration of neutrophils within lamina propria and crypt epithelium, "cryptitis") graded 0-2; (g) degree of transmural inflammation graded 0-2; and (h) degree of mucosal surface erosion up to total surface ulceration graded 0-2. Total histopathologic score was determined from the sum of the scores for each parameter to reflect the overall degree of inflammation within the specimen. Additional sections were stained by immunoperoxidase using primary antibodies directed against CD3 (Dako, A0452) and FOXP3 (eBioscience, 13-5773) (13).

Luciferase assays. $293 \mathrm{~T}$ cells (ATCC) were cultured $\left(37^{\circ} \mathrm{C}, 5 \% \mathrm{CO}_{2}\right)$ in RPMI-1640 plus 10\% heat-inactivated FBS, penicillin and streptomycin, and transfected with NFAT-IL-2 promoter luciferase reporter, plus NFAT, FOXP3, HDAC3 expression vectors or empty vector, using Lipofectamine 2000 (Invitrogen) when cells were 80\%-90\% confluent. After 48 hours, cells were treated with PMA $(6 \mathrm{ng} / \mathrm{ml})$ and ionomycin $(1 \mu \mathrm{M})$ for 5-6 hours and luciferase activities of whole-cell lysates analyzed with a dual-luciferase reporter assay kit (Promega).

ELISA. Supernatant IL-2, IL-6, and IL-17A levels were determined by ELISA (88-7371-22, eBioscience).

ChIP assays. Teffs or Tregs were fixed with $1 \%$ formaldehyde and fragmented by sonication. Chromatin was immunoprecipitated with antibodies directed against trimethylated histone H3 (H3K9, Cell Signaling Technology, \#9754S; H3K27, Cell Signaling Technology, \#9756S), HDAC3 (Cell Signaling Technology, clone D1H2, \#4499), and acetylated histone H3 (Cell Signaling Technology, clone C5B11, \#9649). The resultant DNA was purified and used for realtime PCR with a Step-One system (Applied Biosystems). Il2 primer sets were as reported previously (58); primers for $I l 6$ were forward: GGGCTAGCCTCAAGGATGACT and reverse: GCATCGAAAGAATCACAACTAGGA; and primers for $I l 17$ were forward: TTCCCTTCTCATCCCTCATC and reverse: GAAAGGCTTGATACCGAA.

HDAC3 viral transduction. Plasmid MinR-1 vector containing HDAC3 construct (MinR1-HDAC3) was generated from a pCMVSport6 expression vector containing murine HDAC3 (pCMV-Sport6Mbd2, MMM 1013-9200215, OpenBiosystems). The HDAC3 cDNA - cut from pCMV-Sport6 using NotI (R0189S, New England Biolabs) and SalI (R0138S, New England Biolabs) and with a blunt end added using DNA polymerase I Klenow (M0210S, New England Biolabs) in the presence of dNTPs - was ligated into the MinR-1 vector using T4 ligase (203003, Stratagene), and plasmid sequence verified. Retroviruses were generated by cotransfection of MinR1-HDAC3 or parental MinR1 vector with pCLeco (Invitrogen) helper plasmid into the 293T Phoenix ecotropic packaging cell line (ATCC) using Lipofectamine 2000 (11668-019, Invitrogen). Supernatants were collected and used to infect purified Tregs isolated with magnetic beads. Isolated Tregs were stimulated with CD3 and CD28 mAbs $(2 \mu \mathrm{g} / \mathrm{ml})$ plus $100 \mathrm{U} / \mathrm{ml}$ mouse IL-2. Activated Tregs were infected with 48-hour viral supernatants harvested from the transfected Phoenix cells, cultured at $37^{\circ} \mathrm{C}$ with $5 \% \mathrm{CO}_{2}$ for 24 hours, and used in suppression assays or for RNA extraction.
Microarray and real-time qPCR. RNA was isolated using RNeasy kits (QIAGEN), and RNA integrity and quantity were analyzed by NanoDrop ND-1000 and Nanochip 2100 Bioanalyzer (Agilent Technologies). Microarray experiments were performed using whole mouse genome oligoarrays (Mouse430a, Affymetrix) and array data analyzed using MAYDAY 2.12 software (59). Array data were subjected to robust multiarray average (RMA) normalization and analyzed using Student's $t$ test. Only data with a false discovery rate-adjusted $P$ value of less than 0.05 and at least $2 \times$ differential expression were included in the analysis. Data underwent $z$-score transformation for display. The microarray data were deposited in the Gene Expression Omnibus database (GEO accession number GSE45952). Expression of individual genes was verified by qPCR. RNA was reverse transcribed to cDNA (Applied Biosystems) and qPCR performed using Taqman primer and probe sets; data were normalized to endogenous 18s rRNA, and relative expression was determined by the formula $2^{-\mathrm{ACT}}$.

Immunoprecipitation and Western blotting. Proteins were isolated using RIPA solution plus protease inhibitor (Sigma-Aldrich). Pull-down antibody was incubated with pre-cleared samples for 2 hours at $4^{\circ} \mathrm{C}$, then overnight with protein $\mathrm{G}$ agarose. Western blots were performed using anti-FLAG and anti-FOXP3 Abs (Cell Signaling Technology).

Confocal microscopy. Cell cytospins were fixed, permeabilized with $0.2 \%$ Triton-100, blocked with normal goat serum for 2 hours,

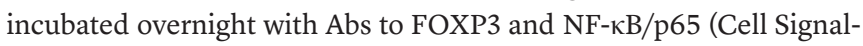
ing Technology, clone C22B4, \#4764) diluted in 0.2\% Triton-X100, washed, stained with secondary anti-rabbit IgG (Cell Signaling Technology, \#7074), and analyzed by confocal microscopy.

Statistics. Data were analyzed using GraphPad Prism 5.0d software. Data are presented as mean \pm SD unless specified otherwise. Measurements between two groups were done with a 2-tailed Student's $t$ test if data were normally distributed or Mann-Whitney $U$ unpaired test when the populations were not normally distributed. Groups of 3 or more were analyzed by 1-way ANOVA with corresponding Tukey's multiple comparison test if normally distributed or the Kruskal-Wallis with Dunn's multiple comparison test if not. Graft survival was evaluated with Kaplan-Meier followed by log-rank test. $P<0.05$ was considered significant.

Study approval. Animal studies were approved by the Institutional Animal Care and Use Committee of the Children's Hospital of Philadelphia (protocols 2013-6-561 and 14-000746).

\section{Acknowledgments}

This work was supported by grants from the NIH to W.W. Hancock (AI073489 and AI095276) and to U.H. Beier (AI095353).

Address correspondence to: Wayne W. Hancock, Division of Transplant Immunology, Department of Pathology and Laboratory Medicine, Children's Hospital of Philadelphia, 3615 Civic Ctr. Blvd., Philadelphia, Pennsylvania 19104, USA. Phone: 215. 590.8709; E-mail: whancock@mail.med.upenn.edu.
1. Samstein RM, et al. Foxp3 exploits a pre-existent enhancer landscape for regulatory $\mathrm{T}$ cell lineage specification. Cell. 2012;151(1):153-166.

2. Rudra D, et al. Transcription factor Foxp3 and its protein partners form a complex regulatory network. Nat Immunol. 2012;13(10):1010-1019.
3. Shevach EM. Biological functions of regulatory T cells. Adv Immunol. 2011;112:137-176.

4. Ohkura N, Kitagawa Y, Sakaguchi S. Development and maintenance of regulatory T cells. Immunity. 2013;38(3):414-423.

5. Riley JL, June CH, Blazar BR. Human T regula- tory cell therapy: take a billion or so and call me in the morning. Immunity. 2009;30(5):656-665.

6. Sakaguchi S, Vignali DA, Rudensky AY, Niec RE, Waldmann H. The plasticity and stability of regulatory T cells. Nat Rev Immunol. 2013;13(6):461-467.

7. Marek-Trzonkowska N, et al. Administration of 
$\mathrm{CD} 4{ }^{+} \mathrm{CD} 25^{\text {high }} \mathrm{CD} 127^{-}$regulatory $\mathrm{T}$ cells preserves $\beta$-cell function in type 1 diabetes in children. Diabetes Care. 2012;35(9):1817-1820.

8. Marek $\mathrm{N}$, et al. The time is crucial for ex vivo expansion of T regulatory cells for therapy. Cell Transplant. 2011;20(11):1747-1758.

9. Trzonkowski P, et al. First-in-man clinical results of the treatment of patients with graft versus host disease with human ex vivo expanded CD4 $\mathrm{CD} 25^{+} \mathrm{CD} 127^{-} \mathrm{T}$ regulatory cells. Clin Immunol. 2009;133(1):22-26.

10. Tao R, et al. Deacetylase inhibition promotes the generation and function of regulatory T cells. Nat Med. 2007;13(11):1299-1307.

11. Liu Y, et al. Inhibition of $\mathrm{p} 300$ impairs Foxp $3^{+} \mathrm{T}$ regulatory cell function and promotes antitumor immunity. Nat Med.2013;19(9):1173-1177.

12. Wang L, de Zoeten EF, Greene MI, Hancock WW. Immunomodulatory effects of deacetylase inhibitors: therapeutic targeting of $\mathrm{FOXP}^{+}$regulatory $\mathrm{T}$ cells. Nat Rev Drug Discov. 2009;8(12):969-981.

13. de Zoeten EF, Wang L, Sai H, Dillmann WH, Hancock WW. Inhibition of HDAC9 increases $T$ regulatory cell function and prevents colitis in mice. Gastroenterology. 2010;138(2):583-594.

14. Beier UH, Wang L, Han R, Akimova T, Liu Y, Hancock WW. Histone deacetylases 6 and 9 and sirtuin-1 control Foxp $3^{+}$regulatory $\mathrm{T}$ cell function through shared and isoform-specific mechanisms. Sci Signal. 2012;5(229):ra45.

15. Lahm A, et al. Unraveling the hidden catalytic activity of vertebrate class IIa histone deacetylases. Proc Natl Acad Sci US A. 2007;104(44):17335-17340.

16. Fischle W, Dequiedt F, Fillion M, Hendzel MJ, Voelter W, Verdin E. Human HDAC7 histone deacetylase activity is associated with HDAC3 in vivo. J Biol Chem. 2001;276(38):35826-35835.

17. Gregoire S, et al. Histone deacetylase 3 interacts with and deacetylates myocyte enhancer factor 2 . Mol Cell Biol. 2007;27(4):1280-1295.

18. Fischle W, et al. Enzymatic activity associated with class II HDACs is dependent on a multiprotein complex containing HDAC3 and SMRT/ N-CoR. Mol Cell. 2002;9(1):45-57.

19. Guenther MG, Barak O, Lazar MA. The SMRT and $\mathrm{N}-\mathrm{CoR}$ corepressors are activating cofactors for histone deacetylase 3. Mol Cell Biol. 2001;21(18):6091-6101.

20. Zheng Y, Josefowicz SZ, Kas A, Chu TT, Gavin MA, Rudensky AY. Genome-wide analysis of Foxp3 target genes in developing and mature regulatory T cells. Nature. 2007;445(7130):936-940.

21. Zheng Y, Josefowicz S, Chaudhry A, Peng XP, Forbush K, Rudensky AY. Role of conserved non-coding DNA elements in the Foxp3 gene in regulatory T-cell fate. Nature. 2010;463(7282):808-812.

22. Wang L, et al. Foxp $3^{+} \mathrm{T}$-regulatory cells require DNA methyltransferase 1 expression to prevent development of lethal autoimmunity. Blood. 2013;121(18):3631-3639.

23. Guenther MG, Lane WS, Fischle W, Verdin E, Lazar MA, Shiekhattar R. A core SMRT corepressor complex containing HDAC3 and TBL1, a
WD40-repeat protein linked to deafness. Genes Dev. 2000;14(9):1048-1057.

24. Li J, et al. Both corepressor proteins SMRT and $\mathrm{N}-\mathrm{CoR}$ exist in large protein complexes containing HDAC3. EMBO J. 2000;19(16):4342-4350.

25. Wen YD, et al. The histone deacetylase-3 complex contains nuclear receptor corepressors. Proc Natl Acad Sci U S A. 2000;97(13):7202-7207.

26. Watson PJ, Fairall L, Santos GM, Schwabe JW. Structure of HDAC3 bound to co-repressor and inositol tetraphosphate. Nature. 2012;481(7381):335-340.

27. Arrar M, Turnham R, Pierce L, de Oliveira CA, McCammon JA. Structural insight into the separate roles of inositol tetraphosphate and deacetylase-activating domain in activation of histone deacetylase 3. Protein Sci. 2013;22(1):83-92.

28. You SH, Lim HW, Sun Z, Broache M, Won KJ, Lazar MA. Nuclear receptor co-repressors are required for the histone-deacetylase activity of HDAC3 in vivo. Nat Struct Mol Biol. 2013;20(2):182-187.

29. Lu L, et al. Role of SMAD and non-SMAD signals in the development of Th17 and regulatory T cells. J Immunol. 2010;184(8):4295-4306.

30. Takimoto $\mathrm{T}$, et al. Smad 2 and $\mathrm{Smad} 3$ are redundantly essential for the TGF- $\beta$-mediated regulation of regulatory $\mathrm{T}$ plasticity and Th1 development. J Immunol. 2010;185(2):842-855.

31. Chen L, Fischle W, Verdin E, Greene WC. Duration of nuclear NF- $\mathrm{kB}$ action regulated by reversible acetylation. Science. 2001;293(5535):1653-1657.

32. Kiernan R, et al. Post-activation turn-off of NF- $\mathrm{kB}$-dependent transcription is regulated by acetylation of p65. J Biol Chem. 2003;278(4):2758-2766

33. Knutson SK, Chyla BJ, Amann JM, Bhaskara S, Huppert SS, Hiebert SW. Liver-specific deletion of histone deacetylase 3 disrupts metabolic transcriptional networks. EMBO J. 2008;27(7):1017-1028.

34. Sun Z, Feng D, Everett LJ, Bugge A, Lazar MA. Circadian epigenomic remodeling and hepatic lipogenesis: lessons from HDAC3. Cold Spring Harb Symp Quant Biol. 2011;76:49-55.

35. Montgomery RL, et al. Maintenance of cardiac energy metabolism by histone deacetylase 3 in mice. J Clin Invest. 2008;118(11):3588-3597.

36. Sun Z, et al. Diet-induced lethality due to deletion of the Hdac3 gene in heart and skeletal muscle. J Biol Chem. 2011;286(38):33301-33309.

37. Razidlo DF, et al. Histone deacetylase 3 depletion in osteo/chondroprogenitor cells decreases bone density and increases marrow fat. PLoS One. 2010;5(7):e11492.

38. McQuown SC, et al. HDAC3 is a critical negative regulator of long-term memory formation. $\mathrm{J} \mathrm{Neu}$ rosci. 2011;31(2):764-774.

39. Chen X, et al. Requirement for the histone deacetylase Hdac3 for the inflammatory gene expression program in macrophages. Proc Natl Acad Sci U S A. 2012;109(42):E2865-2874.

40. Thapa $P$, et al. The transcriptional repressor NKAP is required for the development of iNKT cells. Nat Commun. 2013;4:1582.
41. Summers AR, et al. HDAC3 is essential for DNA replication in hematopoietic progenitor cells. JClin Invest. 2013;123(7):3112-3123.

42. Wong RH, Chang I, Hudak CS, Hyun S, Kwan HY, Sul HS. A role of DNA-PK for the metabolic gene regulation in response to insulin. Cell. 2009;136(6):1056-1072.

43. Yuan Z, Peng L, Radhakrishnan R, Seto E. Histone deacetylase 9 (HDAC9) regulates the functions of the ATDC (TRIM29) protein. J Biol Chem. 2010;285(50):39329-39338.

44. Schuetz A, et al. Human HDAC7 harbors a class IIa histone deacetylase-specific zinc binding motif and cryptic deacetylase activity. J Biol Chem. 2008;283(17):11355-11363.

45. Bradner JE, et al. Chemical phylogenetics of histone deacetylases. Nat Chem Biol.2010;6(3):238-243.

46. Mihaylova MM, et al. Class IIa histone deacetylases are hormone-activated regulators of FOXO and mammalian glucose homeostasis. Cell. 2011;145(4):607-621.

47. Schapira M. Structural biology of human metal-dependent histone deacetylases. Handb Exp Pharmacol. 2011;206:225-240.

48. Wing JB, Sakaguchi S. Foxp3(+) T(reg) cells in humoral immunity. Int Immunol. 2014;26(2):61-69.

49. Reinhardt A, et al. CCR7-mediated migration in the thymus controls $\gamma \Delta$ T-cell development. Eur J Immunol. 2014;44(5):1320-1329.

50. Cyster JG, Schwab SR. Sphingosine-1-phosphate and lymphocyte egress from lymphoid organs. Annu Rev Immunol. 2012;30:69-94.

51. Sun Z, et al. Deacetylase-independent function of HDAC3 in transcription and metabolism requires nuclear receptor corepressor. Mol Cell. 2013;52(6):769-782.

52. Li B, et al. FOXP3 interactions with histone acetyltransferase and class II histone deacetylases are required for repression. Proc Natl Acad Sci U S A. 2007;104(11):4571-4576.

53. Lee PP, et al. A critical role for Dnmt1 and DNA methylation in $\mathrm{T}$ cell development, function, and survival. Immunity. 2001;15(5):763-774.

54. Rubtsov YP, et al. Regulatory T cell-derived interleukin-10 limits inflammation at environmental interfaces. Immunity. 2008;28(4):546-558.

55. Bhaskara S, et al. Deletion of histone deacetylase 3 reveals critical roles in $S$ phase progression and DNA damage control. Mol Cell. 2008;30(1):61-72.

56. Wang L, Han R, Hancock WW. Programmed cell death 1 (PD-1) and its ligand PD-L1 are required for allograft tolerance. Eur J Immunol. 2007;37(10):2983-2990.

57. Aranda R, et al. Analysis of intestinal lymphocytes in mouse colitis mediated by transfer of $\mathrm{CD}^{+}, \mathrm{CD} 45 \mathrm{RBhigh} \mathrm{T}$ cells to SCID recipients. JImmunol. 1997;158(7):3464-3473.

58. Northrop JK, Thomas RM, Wells AD, Shen H. Epigenetic remodeling of the IL-2 and IFN- $\gamma$ loci in memory CD8 T cells is influenced by CD $4 \mathrm{~T}$ cells. J Immunol. 2006;177(2):1062-1069.

59. Battke F, Symons S, Nieselt K. Mayday - integrative analytics for expression data. BMC Bioinformatics. 2010;11:121. 